\title{
Convex duality for stochastic singular control problems
}

\author{
P. Bank \\ Technische Universität Berlin \\ Institut für Mathematik \\ Straße des 17. Juni 136, 10623 Berlin, Germany \\ H. Kauppila \\ Columbia University in the City of New York \\ Department of Mathematics \\ 2990 Broadway, New York, NY 10027
}

August 28, 2018

\begin{abstract}
We develop a general theory of convex duality for certain singular control problems, taking the abstract results by Kramkov and Schachermayer [20] for optimal expected utility from nonnegative random variables to the level of optimal expected utility from increasing, adapted controls. The main contributions are the formulation of a suitable duality framework, the identification of the problem's dual functional as well as the full duality for the primal and dual value functions and their optimizers. The scope of our results is illustrated by an irreversible investment problem and the Hindy-Huang-Kreps utility maximization problem for incomplete financial markets.
\end{abstract}

Keywords: Convex duality, singular control, utility maximization, incomplete markets, irreversible investment.

JEL Classification: G11, G12, C61.

AMS Subject Classification (2010): 93E20, 91G80, 46N10, 91B08. 


\section{Introduction}

A typical stochastic optimal control problem is formulated by specifying how the dynamics of a given system can be influenced by a controller to optimize some performance criterion. In classical stochastic control the controller directly affects the coefficients which govern the system's dynamics, but has no direct influence on the system's state itself. In singular control problems, the controller can, by contrast, directly change the state of the controlled system at any time in a fully scalable way, from infinitesimal to large jumps.

Ever since the seminal work on such singular problems by Beneš et al. [6] the most commonly used approach is to consider Markovian systems and use dynamic programming to derive and then solve the problem's HamiltonJacobi-Bellman equation which comes in the form of a free-boundary value problem. Alternatively, one can resort to versions of Pontryagin's maximum principle as first discussed for stochastic singular control by Cadenillas and Haussmann [9]. In either case, the derived mathematical concepts do not immediately solve the problem, but merely help to describe some of the solution's properties. A key challenge is then to work out this description as neatly as possible. Clearly, this task is made easier when, as we shall assume, control can only be exerted in one direction. Problems of this type include the monotone follower of, e.g., Karatzas and Shreve [17], some irreversible investment problems as discussed in Section 4.1, but also optimal investment and consumption problems with so-called Hindy-Huang-Kreps utilities which we cover in Section 4.2.

All of these problems can be cast as maximization problems for functionals $\mathbb{U}$ of the form

$$
\mathbb{U}(C)=\mathbb{E} \int_{0}^{\infty} U_{t}\left(C_{t}\right) d \mu_{t}
$$

where $C$ is from the class $\mathscr{C}$ of nonnegative, increasing, left-continuous adapted controls, $U_{t}\left(C_{t}\right)$ describes the predictable utility obtained at time $t \geq 0$ from the cumulative control $C_{t}$ and where the optional random measure $\mu$ describes the weights assigned to utilities at different times.

It is the purpose of this paper to develop a theory of convex duality for singular control problems with target functionals of the above type. Indeed, under natural assumptions on $U$ and $\mu$, our first main Theorem 3.1 establishes the Legendre-Fenchel duality of the functional $\mathbb{U}$. For this we introduce the class $\mathscr{D}$ of nonnegative, decreasing, right-continuous processes $D$ as dual 
variables with the pairing

$$
\mathbb{E}\langle C, D\rangle=\mathbb{E} \int_{[0, \infty)} D_{t} d C_{t}
$$

and we show that the Legendre-Fenchel transform

$$
\mathbb{V}(D)=\sup _{C \in \mathscr{C}}\{\mathbb{U}(C)-\mathbb{E}\langle C, D\rangle\}
$$

coincides with the functional

$$
\mathbb{V}(D)=\inf _{\delta \in \dot{\mathscr{D}}(D)} \mathbb{E} \int_{0}^{\infty} V_{t}\left(\delta_{t}\right) d \mu_{t}
$$

where $\dot{D}(D)$ is a certain class of optional processes associated with $D$ and where $V_{t}$ denotes the classical Legendre-Fenchel transform of $U_{t}$. Moreover, we show that the minimizer for $\mathbb{V}(D)<\infty$ can be constructed in terms of a certain envelope process of the form $\breve{D}=\int^{\infty} U^{\prime}\left(C^{D}\right) d \mu$ with $C^{D} \in \mathscr{C}$ which is characterized uniquely by

$$
\mathbb{E}\left[\breve{D}_{t} \mid \mathscr{F}_{t}\right] \leq \mathbb{E}\left[D_{t} \mid \mathscr{F}_{t}\right] \text { for all } t \geq 0
$$

with '=' holding true whenever $C^{D}$ is increasing. We thus obtain a full characterization of the maximizers for

$$
\mathbb{U}(C)-\mathbb{E}\langle C, D\rangle=\mathbb{E} \int_{0}^{\infty} U_{t}\left(C_{t}\right) d \mu_{t}-\mathbb{E} \int_{0}^{\infty} D_{t} d C_{t},
$$

a general form, for instance, of irreversible investment problems as described in Section 4.1.

For the treatment of constrained problems such as the Hindy-HuangKreps optimal investment and consumption problem of Section 4.2 we formulate the abstract utility maximization problem with value function

$$
\mathbf{u}(x)=\sup _{C \in \mathscr{C}(x)} \mathbb{U}(C)
$$

where, for $x>0$, controls are constrained to lie in $\mathscr{C}(x) \subset \mathscr{C}$. This is assumed to be a convex class of feasible controls for which a polar relation with sets $\mathscr{D}(y) \subset \mathscr{D}, y>0$, can be established. This leads to the dual problems with value

$$
\mathbf{v}(y)=\inf _{D \in \mathscr{D}(y)} \mathbb{V}(D)
$$


for $y>0$.

The celebrated papers by Kramkov and Schachermayer [20, 21] develop convex duality for similarly abstract utility maximization problems where utility is obtained at a single point in time, which in our setting amounts to the choice of $\mu$ as a Dirac measure at some point $T>0$. This leads to the obvious challenge to develop a similar convex duality theory for our singular framework. This challenge is taken up by our second main result, Theorem 3.2. While our proof of this result follows to some extent the very useful blue-print laid out by Kramkov and Schachermayer [20], there are a number of novel obstacles to overcome along the way. These are a consequence of our central constraint of increasing adapted controls which in the setting of Kramkov and Schachermayer corresponds to the considerably simpler restriction to nonnegative $\mathscr{F}_{T}$-measurable random variables. This also distinguishes our work from Karatzas and Žitković [18] who consider utility from consumption at nonnegative rates, i.e., without the monotonicity constraint of our singular control set.

Specifically, a first key difference is in the structure of the LegendreFenchel transform of the utility functional under consideration: For Kramkov and Schachermayer's $C \mapsto \mathbb{E} U_{T}\left(C_{T}\right)$ the dual functional is simply $D \mapsto$ $\mathbb{E} V_{T}\left(D_{T}\right)$ whereas the dual $\mathbb{V}$ of our functional $\mathbb{U}$ involves an infimum. As a consequence, the connection between the dual value $\mathbb{V}(D)$ and the dual variable $D$ is not as straight forward as in [20] but has to be described by our envelope process $\breve{D}$. Also, the process $C^{D}$ which is conjugate to $D$ in the Legendre-Fenchel duality cannot be directly written in terms of $D$, by contrast to [20] where one merely has to invert $U_{T}^{\prime}\left(C_{T}^{D}\right)=D_{T}$. In addition, the dual problem is not strictly convex anymore, a property which is needed for some of the arguments in Kramkov and Schachermayer [20]. As a remedy, we introduce a subclass of $\mathscr{D}(y)$ which is sufficiently large to include the solutions to the dual problem, but small enough to ensure strict convexity of $\mathbb{V}$ on this subclass. This allows us to establish the continuous dependence of certain solutions to the dual problem on the Lagrange parameter $y$. The final challenge is then to show that the corresponding candidate solutions for the primal problem are indeed feasible for the larger class of all dual variables $\mathscr{D}(y)$. Here, we have to resort to the general Legendre-Fenchel duality between $\mathbb{U}$ and $\mathbb{V}$ developed in our first main result. Finally, the notion of reasonable asymptotic elasticity identified by [20] as a key assumption for general well-posedness of utility maximization problems has to be adapted to account for the possibly very different utility functions $U_{t}$ at different 
time points $t \geq 0$. In fact, in line with, e.g., Bouchard and Pham [8] and Žitković [25] we do allow for time- and scenario-dependent utility functions and a stochastic clock which allows us to include the finite time horizon case in the infinite time horizon formulation in a simple manner; see the end of Section 4.2.

The paper is organized as follows. In Section 2 we introduce the class of controls $\mathscr{C}$ and the space of dual variables $\mathscr{D}$ along with the assumptions and definition of our utility functional $\mathbb{U}$ and its dual $\mathbb{V}$. Section 3 is devoted to the presentation of our main duality results, Theorems 3.1 and 3.2. Section 4 illustrates these findings by a general irreversible investment problem and by the optimal consumption problem of Hindy, Huang, and Kreps. Section 5 contains the proofs our main theorems. Appendix A gives the construction of our envelope process $\breve{D}$. Appendix B discusses Žitković [26]'s notion of convex compactness in the new context of our class of controls $\mathscr{C}$ and provides a minimax theorem compatible with this generalized notion of compactness.

\section{Controls and their performance measure}

We start by describing the control set $\mathscr{C}$ and its dual $\mathscr{D}$ as well as our target utility functional $\mathbb{U}$ along with a dual functional $\mathbb{V}$. As usual, we let $(\Omega, \mathscr{F}, \mathbb{F}, \mathbb{P})$ denote throughout a filtered probability space describing a controller's beliefs $\mathbb{P}$ about future events $\mathscr{F}$ along with his information flow $\mathbb{F}=\left(\mathscr{F}_{t}\right)_{t \geq 0}$, a complete, right-continuous filtration where $\mathscr{F}_{0}$ is generated by the $\mathbb{P}$-null sets.

\subsection{Controls and their duals}

The set of conceivable controls will be given by the class $\mathscr{C}$ of predictable processes $C: \Omega \times[0, \infty] \rightarrow[0, \infty]$ with non-decreasing, left-continuous paths starting from $C_{0}=0$. As usual exercising control incurs costs which will be described by dual variables. A convenient set of such dual variables will turn out to be the class $\mathscr{D}$ of all $\mathscr{F} \otimes \mathscr{B}([0, \infty])$-measurable processes $D$ : $\Omega \times[0, \infty] \rightarrow[0, \infty]$ with non-increasing, right-continuous paths ending in $D_{\infty}=0$. Indeed, for any $C \in \mathscr{C}$ and $D \in \mathscr{D}$ we can define

$$
\langle C, D\rangle \triangleq \int_{[0, \infty)} D_{t} d C_{t}=-\int_{(0, \infty]} C_{t} d D_{t},
$$


which yields the pairing

$$
\mathbb{E}\langle C, D\rangle=\mathbb{E} \int_{[0, \infty)} D_{t} d C_{t}=-\mathbb{E} \int_{(0, \infty]} C_{t} d D_{t} \in[0, \infty] .
$$

Observe that the above identities are to be understood and hold with the following conventions regarding the integration with respect to $C \in \mathscr{C}$ and $D \in \mathscr{D}:$

- $d C$ and $d D$ do not charge the intervals (inf $\left.\left\{t \geq 0: C_{t}=\infty\right\}, \infty\right]$ and $\left[0, \sup \left\{t \geq 0: D_{t}=\infty\right\}\right)$, respectively;

- the integration with respect to $d C$ is carried out taking into account a point mass of size $C_{0+} \triangleq \lim _{t \downarrow 0} C_{t}$ at 0 and the integration with respect to $d D$ assumes a point mass $D_{\infty_{-}} \triangleq \lim _{t \uparrow \infty} D_{t}$ at $\infty$;

- we let $0 \cdot \infty \triangleq 0$ should an integrand be zero where the integrator puts an infinite point mass.

Finally, we note that both $\mathscr{C}$ and $\mathscr{D}$ can be endowed with the metric which for two $\mathscr{F} \otimes \mathscr{B}([0, \infty])$-measurable processes $A, B$ assigns the distance

$$
\operatorname{dist}(A, B) \triangleq \mathbb{E} \int_{0}^{\infty}\left|h\left(A_{t}\right)-h\left(B_{t}\right)\right| d \mu_{t}
$$

where $h$ is any homeomorphism $[-\infty, \infty] \rightarrow[0,1]$. With respect to this distance the pairing (1) is lower-semicontinuous in each of its factors; see Lemma B.1.

\subsection{Utilities and their conjugates}

The performance of controls will be measured by the utilities they provide at each time, weighted with the controller's time preferences.

Assumption 2.1. The controller's time preferences are described by an optional random measure $\mu$ on $[0, \infty)$ without atoms, full support and finite expected total mass $\mathbb{E} \mu([0, \infty))<\infty$.

The controller's utility is specified by a mapping

$$
\begin{aligned}
U: \Omega \times[0, \infty) \times[0, \infty) & \rightarrow[0, \infty) \\
(\omega, t, c) & \mapsto U_{t}(\omega, c)
\end{aligned}
$$

with the following properties: 
1. For any $(\omega, t) \in \Omega \times[0, \infty), U_{t}(\omega,$.$) is continuous, strictly concave and$ strictly increasing from $U_{t}(\omega, 0)=0$ to $U_{t}(\omega, \infty) \triangleq \lim _{c \uparrow \infty} U_{t}(\omega, c) \in$ $[0, \infty]$. Moreover, $U_{t}(\omega,$.$) is continuously differentiable and satisfies the$ Inada conditions

$$
U_{t}^{\prime}(\omega, 0) \triangleq \lim _{c \downarrow 0} U_{t}^{\prime}(\omega, c)=\infty \text { and } U_{t}^{\prime}(\omega, \infty) \triangleq \lim _{c \uparrow \infty} U_{t}^{\prime}(\omega, c)=0 .
$$

2. For any $c \geq 0,(\omega, t) \mapsto U_{t}(\omega, c)$ is predictable with $\mathbb{E} \int_{0}^{\infty} U_{t}(c) d \mu_{t}<\infty$.

3. The asymptotic elasticity of $U$ is uniformly less than one in the sense that there is a constant $\gamma \in(0,1)$ and a predictable process $C^{\gamma} \geq 0$ with $\mathbb{E} \int_{0}^{\infty} U_{t}\left(C_{t}^{\gamma}\right) d \mu_{t}<\infty$ such that for any $(\omega, t) \in \Omega \times[0, \infty)$ we have

$$
\frac{c U_{t}^{\prime}(\omega, c)}{U_{t}(\omega, c)}<\gamma<1 \text { for all } c>C_{t}^{\gamma}(\omega)
$$

A control will provide an expected utility of the form

$$
\mathbb{U}(C) \triangleq \mathbb{E} \int_{0}^{\infty} U_{t}\left(C_{t}\right) d \mu_{t} \in[0, \infty], \quad C \in \mathscr{C} .
$$

Note that in our setting, contrary to what is more commonly assumed, utility $U_{t}\left(C_{t}\right)$ at each time $t \geq 0$ is obtained from the cumulative control $C_{t}$ rather than the current control rate. This turns the optimization problem to be introduced shortly into a singular stochastic control problem. We refer to the illustrations of Section 4 for the motivation and scope of such utility functionals.

Remark 2.2. Let us briefly comment on our preference Assumption 2.1:

1. The first item just requires that time- and scenario-wise the utility function is standard, except for the requirement that utility at zero vanishes. In fact, this comes without loss of generality if $\mathbb{E} \int_{0}^{\infty}\left|U_{t}(0)\right| d \mu_{t}<\infty$ since then we can pass to $\tilde{U} \triangleq U-U(0)$ without changing the utility maximization problem for (4).

2. The predictability requirement in the second item is essentially without loss of generality since we could work with the predictable projection of any non-predictable field $(U(c), c \in[0, \infty))$ without changing (4). This holds because controls are predictable and because time preferences are optional random measures without atoms. 
3. It is well-known from the work of Kramkov and Schachermayer [20] that asymptotic elasticity less than one is necessary to avoid ill-posed utility maximization problems. Their Lemma 6.3 shows that for any $(\omega, t) \in \Omega \times[0, \infty)$, condition (3) is equivalent to

$$
U_{t}(\omega, \lambda c)<\lambda^{\gamma} U_{t}(\omega, c) \text { for all } \lambda>1 \text { and all } c>C_{t}^{\gamma}(\omega) \text {. }
$$

4. Our results will also allow us to treat the case of a possibly finite time horizon given by some stopping time $\tau$; see Remark 4.1 below.

One of the main results in this paper is the convex duality for the functional $\mathbb{U}$ of (4) which will be established in Theorem 3.1 below. For this we need to introduce a dual functional $\mathbb{V}$ on $\mathscr{D}$. This functional will be specified in terms of the classical Legendre-Fenchel transform $V$ of $U$ :

$$
V_{t}(\omega, d) \triangleq \sup _{0 \leq c<\infty}\left\{U_{t}(\omega, c)-c d\right\}, \quad d>0
$$

It is well-known that under the conditions in Assumption $2.1 V_{t}(\omega,$.$) is a$ strictly convex and decreasing function on $(0, \infty)$ with

$V_{t}(\omega, 0) \triangleq \lim _{d \downarrow 0} V_{t}(\omega, d)=U_{t}(\omega, \infty)$ and $V_{t}(\omega, \infty) \triangleq \lim _{d \uparrow \infty} V_{t}(\omega, d)=U_{t}(\omega, 0)=0$

Moreover, $V_{t}(\omega,$.$) is continuously differentiable on (0, \infty)$ and satisfies the Inada conditions

$$
V_{t}^{\prime}(\omega, 0) \triangleq \lim _{d \downarrow 0} V_{t}^{\prime}(\omega, d)=-\infty \text { and } V_{t}^{\prime}(\omega, \infty) \triangleq \lim _{d \uparrow \infty} V_{t}^{\prime}(\omega, d)=0 .
$$

The asymptotic elasticity conditions (3) and (5) can be cast in terms of $V$ as

$$
(1-\gamma)\left(-V^{\prime}(d)\right) d \leq \gamma V(d) \text { for all } 0<d<D^{\gamma}
$$

and

$$
V((1-\varepsilon) d)<(1-\varepsilon)^{\frac{\gamma}{1-\gamma}} V(d) \text { for all } 0<\varepsilon<1,0<d<D^{\gamma}
$$

with the same $\gamma \in(0,1)$ as before and $D^{\gamma} \triangleq U^{\prime}\left(C^{\gamma}\right)$; see Lemma 6.3 in Kramkov and Schachermayer [20]. Finally, along with $U$ also $V$ is predictable and we have the following conjugacy relations: 
1. In addition to (6), we also have

$$
U_{t}(\omega, c)=\inf _{0 \leq d<\infty}\left\{V_{t}(\omega, d)+c d\right\}, \quad c>0 .
$$

2. The supremum in (6) is attained at $c=-V_{t}^{\prime}(\omega, d)$.

3. The infimum in (9) is attained at $d=U_{t}^{\prime}(\omega, c)$.

In fact, the identities in items 2. and 3. are equivalent.

We now can introduce the dual functional

$$
\mathbb{V}(D) \triangleq \inf _{\delta \in \dot{D}(D)} \mathbb{E} \int_{0}^{\infty} V_{t}\left(\delta_{t}\right) d \mu_{t} \in[0, \infty], \quad D \in \mathscr{D},
$$

with

$$
\dot{\mathscr{D}}(D) \triangleq\left\{\delta \geq 0 \text { optional : }{ }^{o}\left(\int^{\infty} \delta d \mu\right) \leq{ }^{o} D\right\}
$$

where we used the notation ${ }^{\circ} X$ for the optional projection of any $\mathscr{F} \otimes$ $\mathscr{B}([0, \infty])$-measurable process $X \geq 0$.

\section{Main results}

\subsection{Legendre-Fenchel duality for utility functionals}

For the statement of our duality theorem for $\mathbb{U}$ and $\mathbb{V}$ of (4) and (10) we have to introduce for any dual process $D \in \mathscr{D}$ a special envelope process $\breve{D}$ of the form

$$
\breve{D}_{t}=\int_{t}^{\infty} U^{\prime}\left(C^{\breve{D}}\right) d \mu, t \geq 0, \text { for some } C^{\breve{D}} \in \mathscr{C}
$$

which satisfies $\mathbb{P}$-almost surely

$$
{ }^{o} \breve{D}_{t} \leq{ }^{o} D_{t} \text { for any } t \geq 0 \text {, with "=" if } d C_{t}^{\breve{D}}>0 \text {. }
$$

Here, we follow the convention that, for $C \in \mathscr{C}$, we write $d C_{t}>0$ iff $t$ is a point of increase for $C$ in the sense that $C_{t}<C_{s+t}$ for any $s>0$. We refer to Lemma A.1 of Appendix A for existence and uniqueness up to indistinguishability of such an envelope process. 
Note that the paths of such an envelope process $\breve{D}$ are absolutely continuous with respect to $\mu$. We choose

$$
\dot{\check{D}} \triangleq-U^{\prime}\left(C^{\breve{D}}\right)
$$

for the corresponding density which is then uniquely determined up to indistinguishability because so is the process $C^{\breve{D}} \in \mathscr{C}$ with (12) and (13). Observe, that, conversely, we can then write $C^{\breve{D}}=-V^{\prime}(-\dot{\breve{D}})$ by the conjugacy relations between $U$ and $V$ recalled above.

We now can state our first main result as follows:

Theorem 3.1. Under Assumption 2.1 the following assertions hold:

1. The functionals $\mathbb{U}$ of (4) and $\mathbb{V}$ of (10) are conjugate to each other in the sense that we have

$$
\mathbb{U}(\hat{C})=\inf _{\mathbb{V}(D)<\infty}\{\mathbb{V}(D)+\mathbb{E}\langle\hat{C}, D\rangle\} \text { for any } \hat{C} \in \mathscr{C}
$$

and

$$
\mathbb{V}(\hat{D})=\sup _{\mathbb{U}(C)<\infty}\{\mathbb{U}(C)-\mathbb{E}\langle C, \hat{D}\rangle\} \text { for any } \hat{D} \in \mathscr{D}
$$

2. If finite, the infimum in (15) is attained for precisely those $D \in \mathscr{D}$ whose (joint) envelope process $\breve{D}$ with (12) and (13) is given by

$$
\dot{\breve{D}}=-U^{\prime}(\hat{C}) \text {. }
$$

3. If finite, the supremum in (16) is attained exactly for

$$
\hat{C}=-V^{\prime}(-\dot{\breve{D}}) \in \mathscr{C}
$$

where $\breve{D}$ is the envelope process of $\hat{D}$ characterized by (12) and (13) with $D \triangleq \hat{D}$.

\subsection{Convex duality for an abstract utility maximiza- tion problem}

Let us now formulate an abstract utility maximization problem in a similar way as in the approach for utility from terminal wealth by Kramkov and Schachermayer [20]. To this end we consider $\mathscr{C}(1) \subset \mathscr{C}$ and $\mathscr{D}(1) \subset \mathscr{D}$ which are polar with respect to each other in the sense that 
1. For any $C \in \mathscr{C}$, we have $C \in \mathscr{C}(1)$ iff $\mathbb{E}\langle C, D\rangle \leq 1$ for any $D \in \mathscr{D}(1)$.

2. For any $D \in \mathscr{D}$, we have $D \in \mathscr{D}(1)$ iff $\mathbb{E}\langle C, D\rangle \leq 1$ for any $C \in \mathscr{C}(1)$.

To avoid trivialities we also assume

3. $\mathscr{C}(1) \supset\{\mathbf{1}\}$ where $\mathbf{1} \in \mathscr{C}$ denotes the control with $\mathbf{1}_{0}(\omega) \triangleq 0$ and $\mathbf{1}_{t}(\omega) \triangleq 1, t \in(0, \infty], \omega \in \Omega$.

4. $\mathscr{D}(1) \neq\{\mathbf{0}\}$ where $\mathbf{0} \in \mathscr{D}$ is the trivial state-price deflator given by $\mathbf{0}_{t}(\omega) \triangleq 0, t \in[0, \infty], \omega \in \Omega$.

The set $\mathscr{C}(1)$ will play the role of the budget set for wealth $x=1$ and $\mathscr{D}(1)$ can be viewed as a set of state price deflators $D \in \mathscr{D}$ (induced, e.g., by a financial market model) for which, in particular, $\mathbb{E} D_{0}=\mathbb{E}\langle\mathbf{1}, D\rangle \leq y=1$.

To formulate the abstract utility maximization problem and its dual let us put

$$
\mathscr{C}(x) \triangleq x \mathscr{C}(1) \text { for } x>0 \text { and } \mathscr{D}(y) \triangleq y \mathscr{D}(1) \text { for } y>0 .
$$

It is clear that $\mathscr{C}(x)$ and $\mathscr{D}(y)$ inherit the polar relation from $\mathscr{C}(1)$ and $\mathscr{D}(1)$ for any $x, y>0$. By this relation it is also obvious that these sets are convex and solid (i.e., e.g., with $C \in \mathscr{C}(x)$, any $\tilde{C} \in \mathscr{C}$ with $\tilde{C} \leq C$ is also contained in $\mathscr{C}(x))$. Moreover, the lower-semicontinuity of the pairing $\mathbb{E}\langle C, D\rangle$, see Lemma B.1, ensures that $\mathscr{C}(x)$ and $\mathscr{D}(y)$ are closed with respect to convergence in the metric (2).

Finally, let us introduce the value functions

$$
\mathbf{u}(x) \triangleq \sup _{C \in \mathscr{C}(x)} \mathbb{U}(C), \quad x>0
$$

and

$$
\mathbf{v}(y) \triangleq \inf _{D \in \mathscr{D}(y)} \mathbb{V}(D), \quad y>0
$$

Theorem 3.2. Suppose that Assumption 2.1 holds true and assume that $\mathbf{u}(x)<\infty$ for some $x>0$. Then we have:

1. The value functions $\mathbf{u}$ of (19) and $\mathbf{v}$ of (20) are real-valued and conjugate to each other in the sense that

$$
\mathbf{u}(x)=\inf _{y>0}\{\mathbf{v}(y)+x y\} \text { for any } x>0
$$


and

$$
\mathbf{v}(y)=\sup _{x>0}\{\mathbf{u}(x)-x y\} \text { for any } y>0 .
$$

Moreover, $\mathbf{u}$ and $\mathbf{v}$ are continuously differentiable on $(0, \infty)$ and satisfy the Inada conditions

$$
\mathbf{u}^{\prime}(0)=\infty, \mathbf{u}^{\prime}(\infty)=0, \mathbf{v}^{\prime}(0)=-\infty, \mathbf{v}^{\prime}(\infty)=0
$$

In addition, $\mathbf{u}$ and $\mathbf{v}$ are, respectively, strictly concave and strictly convex, and $y$ attains the infimum in (21) iff $x$ attains the supremum in (22) which in turn is equivalent to both

$$
\mathbf{u}^{\prime}(x)=y \text { and } \mathbf{v}^{\prime}(y)=-x .
$$

2. The infimum in the dual problem (20) is attained for any $y>0$. All the minimizers $D$ of (20) have the same envelope process $D^{y} \in \mathscr{D}(y)$ with (12) and (13), and, for $x$ given by (24),

$$
C^{x}=-V^{\prime}\left(-\dot{\breve{D}}^{y}\right) \in \mathscr{C}(x)
$$

attains the supremum in the primal problem (19).

3. The supremum in the primal problem (19) is attained for any $x>0$ at a unique $C^{x} \in \mathscr{C}(x)$ and, for $y$ given by $(24)$,

$$
\dot{\breve{D}}^{y}=-U^{\prime}\left(C^{x}\right)
$$

yields via (12) a $\breve{D}^{y} \in \mathscr{D}(y)$ which attains the infimum in the dual problem (20).

\section{Illustrations}

Let us illustrate the usefulness of Theorems 3.1 and 3.2 by showing how they can be brought to bear on the classical problems of irreversible investment and of optimal consumption and investment. 


\subsection{Irreversible investment}

Consider the manager of a firm who can decide at any point in time $t \geq 0$ whether or not to expand the currently installed capacity of production $C_{t}$. Assuming that installed capacity cannot be reduced in a profitable way amounts to the assumption that $C \in \mathscr{C}$ as introduced in Section 2.1. Let us suppose that the revenues $R_{t}^{C}$ from the firm's production are an increasing function of installed capacity and exhibit decreasing returns to scale. Plainly, it is perfectly reasonable to assume that revenues also depend on the product's price fluctuations and possibly other stochastically evolving market conditions. It thus makes sense to assume that, at time $t \geq 0$, the revenues from a capacity expansion policy $C \in \mathscr{C}$ are given as

$$
R_{t}^{C}(\omega)=U_{t}\left(\omega, C_{t}(\omega)\right)
$$

for some function $U: \Omega \times[0, \infty) \times[0, \infty) \rightarrow[0, \infty)$ as considered in Assumption 2.1. The manager discounts future cash flows at some rate $r=\left(r_{t}\right)_{t \geq 0}$, an optional process with $\int_{0}^{t}\left|r_{s}\right| d s<\infty, t \geq 0$, which we assume to be such that the random measure

$$
\mu(d t) \triangleq e^{-\int_{0}^{t} r_{s} d s} d t
$$

has finite expected mass $\mathbb{E} \mu(0, \infty)<\infty$.

The expected total discounted revenue is then given by

$$
\mathbb{E} \int_{0}^{\infty} e^{-\int_{0}^{t} r_{s} d s} R_{t}^{C} d t=\mathbb{E} \int_{0}^{\infty} U_{t}\left(C_{t}\right) d \mu_{t}=\mathbb{U}(C)
$$

exactly as considered in (4). If we now assume that the (discounted) cost of expanding production capacity by one unit at time is described by a class (D) supermartingale $Z \geq 0$ with $Z_{\infty}=0$ we are led to consider the manager's optimization problem:

$$
\text { Maximize } \mathbb{U}(C)-\mathbb{E} \int_{0}^{\infty} Z_{t} d C_{t} \text { subject to } C \in \mathscr{C} \text {. }
$$

This kind of singular control problem is of great interest in Economics. We refer to Alvarez [1] for a more extensive account of the pertaining literature.

Recalling the Doob-Meyer decomposition $Z=M-A$ into a uniformly integrable martingale $M$ and a predictable increasing process $A$ with $A_{0}=0$, we find that $\hat{D} \triangleq M_{\infty}-A$ is contained in $\mathscr{D}$ and satisfies

$$
\mathbb{E} \int_{0}^{\infty} Z_{t} d C_{t}=\mathbb{E} \int_{0}^{\infty}{ }^{o}\left(M_{\infty}-A_{t}\right) d C_{t}=\mathbb{E}\langle C, \hat{D}\rangle, \quad C \in \mathscr{C} .
$$


By Theorem 3.1, the value of problem (27) is thus given by the dual functional $\mathbb{V}(\hat{D})$ of $(16)$ and, if it is finite, we obtain that the optimal capacity expansion plan is $\hat{C}$ with (18). In particular, an explicit solution to (27) can be given whenever the envelope process $\breve{D}$ associated with $\hat{D}$ can be computed explicitly. We refer to Chiarolla and Ferrari [10], Ferrari [13], Bank and Riedel [5], Bank and Baumgarten [2] for such examples.

\subsection{Hindy-Huang-Kreps utility}

Following the seminal work of Merton [22], the problem of optimal investment and consumption in continuous-time is mostly studied for utility functions which depend on the current consumption rate. This modeling approach was shown by Hindy, Huang, and Kreps (see [16, 14, 15]) to fail to exhibit the economically desirable property of intertemporal substitution: in Merton's setting, slight shifts in the timing of consumption plans may lead to significant changes in the utility associated with these plans. As a remedy, these authors proposed to consider functionals where utility is derived from a level of satisfaction, i.e., a weighted average of past consumption such as

$$
Y_{t}^{\tilde{C}} \triangleq \int_{0}^{t} e^{-\int_{s}^{t} \beta_{u} d u} d \tilde{C}_{s}, \quad t \geq 0
$$

where $\tilde{C} \in \mathscr{C}$ describes the cumulative consumption and where the locally Lebesgue-integrable adapted process $\beta \geq 0$ measures the decay rate of satisfaction. The utility functional to be maximized is then

$$
\tilde{\mathbb{U}}(\tilde{C}) \triangleq \mathbb{E} \int_{0}^{\infty} \tilde{U}\left(Y_{t}^{\tilde{C}}\right) d \mu_{t}
$$

where $\tilde{U}:[0, \infty) \rightarrow \mathbb{R}$ is a strictly concave and increasing utility function of class $C^{1}$ satisfying the Inada conditions $\tilde{U}^{\prime}(0)=\infty$ and $\tilde{U}^{\prime}(\infty)=0 ; \mu$, as before, describes an agent's time-preferences and could, for instance, be specified as $\mu(d t)=e^{-\delta t} d t$ with $\delta>0$.

As usual, the set of consumption plans at the agent's disposal is determined by his investment opportunities. Assuming the mild assumption of no free lunch with vanishing risk we obtain from the celebrated Fundamental Theorem of Asset Pricing of Delbaen and Schachermayer [11, 12] in great generality that this set can be described in the form

$$
\tilde{\mathscr{C}}(x)=\left\{\tilde{C} \in \mathscr{C}: \mathbb{E} \int_{[0, \infty)} Z_{t} d C_{t} \leq x \text { for all } Z \in \mathscr{Z}\right\},
$$


where $x$ denotes the available initial capital and $\mathscr{Z}$ denotes a nonempty set of local martingale deflators, i.e., of $\mathbb{P}$-supermartingales $Z>0$ with $Z_{0}=1$ such that for any wealth process $V$ of an admissible investment strategy the process $Z V$ is a $\mathbb{P}$-supermartingale.

The agent's optimization problem is then to

$$
\text { Maximize } \tilde{\mathbb{U}}(\tilde{C}) \triangleq \mathbb{E} \int_{0}^{\infty} \tilde{U}\left(Y_{t}^{\tilde{C}}\right) d \mu_{t} \text { subject to } \tilde{C} \in \tilde{\mathscr{C}}(x) \text {. }
$$

To transform this into the type of utility maximization treated by our main results in Section 3 consider the bijection

$$
\mathscr{C} \ni \tilde{C} \mapsto C \triangleq\left(\int_{0}^{t} e^{\int_{0}^{s} \beta_{u} d u} d \tilde{C}_{s}\right)_{t \geq 0} \in \mathscr{C}
$$

and let

$$
U_{t}(\omega, c) \triangleq \tilde{U}\left(e^{-\int_{0}^{t} \beta_{u}(\omega) d u} c\right) .
$$

Then the utility functional $\mathbb{U}$ of (4) satisfies

$$
\mathbb{U}(C)=\tilde{U}(\tilde{C}) .
$$

Let us also put

$$
\mathscr{C}(1) \triangleq\{C \in \mathscr{C}: \tilde{C} \text { with }(30) \text { is contained in } \tilde{\mathscr{C}}(1)\}
$$

and consider its polar

$$
\mathscr{D}(1) \triangleq\{D \in \mathscr{D}: \mathbb{E}\langle C, D\rangle \leq 1 \text { for all } C \in \mathscr{C}(1)\}
$$

This latter set is different from $\{\mathbf{0}\}$. Indeed, take any local martingale deflator $Z \in \mathscr{Z}$ and let $Z=M \tilde{D}$ be its multiplicative Doob-Meyer decomposition into a local martingale $M$ and a predictable decreasing process $\tilde{D}$ with $\tilde{D}_{0}=1$. Let $\left(T^{n}\right)_{n=1,2, \ldots}$ be a localizing sequence of stopping times such that each of the stopped supermartingales $Z^{T^{n}}$ (and, thus, each of the stopped local martingales $\left.M^{T^{n}}\right), n=1,2, \ldots$, is of class (D). Observe then that $D_{t}^{n} \triangleq\left(M_{T^{n}} \tilde{D}_{t}\right) e^{-\int_{0}^{t} \beta_{u} d u} 1_{\left[0, T^{n}\right.}(t), t \geq 0$, is contained in $\mathscr{D}$ and

$$
\mathbb{E}\left\langle C, D^{n}\right\rangle=\mathbb{E} \int_{\left[0, T^{n}\right)}{ }^{o}\left(M_{T^{n}} \tilde{D} e^{-\int_{0} \beta_{u} d u}\right)_{t} d C_{t}=\mathbb{E} \int_{\left[0, T^{n}\right)} Z_{t} d \tilde{C}_{t} \leq 1
$$


for any $C \in \mathscr{C}(1)$. Hence, $D^{n} \in \mathscr{D}(1)$ for each $n=1,2, \ldots$ In fact, letting $n \uparrow \infty$ in (31) we find in conjunction with (28):

$$
\mathscr{C}(1)=\{C \in \mathscr{C}: \mathbb{E}\langle C, D\rangle \leq 1 \text { for any } D \in \mathscr{D}(1)\} .
$$

Hence, $\mathscr{C}(1)$ and $\mathscr{D}(1)$ exhibit the polar relations assumed in the beginning of Section 3.2.

It thus follows that we have the convex duality results of Theorem 3.2 for the Hindy-Huang-Kreps-utility maximization problem (29). This generalizes the treatment of the complete market case in [5] to incomplete market models driven by general semimartingales and thus also complements the dynamic programming approach for exponential Levy models with constant relative risk aversion of Benth et al. [7]. In particular, the present paper develops convex duality for optimal consumption with Hindy-Huang-Kreps preferences at a level of generality similar to Kramkov and Schachermayer [20] for utility from terminal wealth and to Karatzas and Žitković [18] for utility from the rate of consumption.

Remark 4.1. It may be worthwhile to observe that our results also cover the finite time horizon case where $\mu$ has support $[0, T]$ for some possibly finite stopping time $T>0$. Indeed, in that case we can instead consider $\bar{\mu}(d t) \triangleq \mu(d t)+1_{(T, \infty)}(t) e^{-t} d t, \bar{U}_{t}(c) \triangleq 1_{[0, T]}(t) U(c)+1_{(T, \infty)}(t) U^{*}(c)$, where $U^{*}:[0, \infty) \rightarrow \mathbb{R}$ is any deterministic utility function satisfying the Inada conditions and having an upper bound $U^{*}(\infty)<\infty$. The budget set will be described by

$$
\overline{\mathscr{D}}(1) \triangleq\left\{D 1_{[0, T)}: D \in \mathscr{D}(1)\right\}
$$

and

$$
\begin{aligned}
\overline{\mathscr{C}}(1) & \triangleq\{C \in \mathscr{C}: \mathbb{E}\langle C, D\rangle \leq 1 \text { for all } D \in \overline{\mathscr{D}}(1)\} \\
& =\left\{C \in \mathscr{C}:\left(C_{t \wedge T}\right)_{t \geq 0} \in \mathscr{C}(1)\right\} .
\end{aligned}
$$

Then $\bar{U}, \bar{\mu}$ satisfy Assumption 2.1 if $U$ does and if $U^{*}$ has asymptotic elasticity less than one. Moreover, $\overline{\mathscr{C}}(1), \overline{\mathscr{D}}(1)$ are polar to each other as requested in Section 3.2 and the consumption plans $C, \bar{C} \in \mathscr{C}$ maximizing

$$
\mathbb{E} \int_{0}^{T} U\left(C_{t}\right) d \mu_{t}, \quad \text { respectively } \mathbb{E} \int_{0}^{\infty} \bar{U}\left(\bar{C}_{t}\right) d \mu_{t}
$$

subject to $C \in \mathscr{C}(x)$, respectively, $\bar{C} \in \overline{\mathscr{C}}(x)$ are actually the same up to time $T$ (when all the optimal $\bar{C}$ jump to $+\infty$ ). 


\section{Proofs of the main results}

\subsection{Proof of Theorem 3.1}

Theorem 3.1 follows readily from Lemmas 5.2 and 5.3 below. These results rely heavily on the following observation:

Lemma 5.1. Suppose Assumption 2.1 holds true. For $D \in \mathscr{D}$ let $\breve{D}$ denote its envelope with (12) and (13) and, recalling (14), consider $\delta^{D} \in \dot{\mathscr{D}}(D)$ of (11) with

$$
C^{D} \triangleq-V^{\prime}\left(\delta^{D}\right) \in \mathscr{C}
$$

Then $\delta^{D}$ attains the infimum in the definition (10) of $\mathbb{V}(D)$ and, if $\mathbb{V}(D)<$ $\infty, \delta^{D}$ is in fact the unique minimizer in $\dot{\mathscr{D}}(D)$, up to modifications on a $\mathbb{P} \otimes \mu$-null set.

Proof. It is immediate from (13) that indeed $\delta^{D} \in \dot{\mathscr{D}}(D)$. Uniqueness of minimizers for (10) is due to the strict convexity of $V$. It thus remains to prove optimality of $\delta^{D}$ for (10). For this it suffices to show that, for $n=1,2, \ldots$,

$$
\mathbb{E} \int_{0}^{\infty} V_{n}(\delta) d \mu \geq \mathbb{E} \int_{0}^{\infty} V_{n}\left(\delta^{D}\right) d \mu \text { for any } \delta \in \dot{\mathscr{D}}(D)
$$

where

$$
V_{n}(d) \triangleq \sup _{0 \leq c \leq n}\{U(c)-c d\}= \begin{cases}U(n)-n d, & 0 \leq d \leq U^{\prime}(n), \\ V(d), & d \geq U^{\prime}(n) .\end{cases}
$$

Indeed, it is readily checked that $V_{n} \geq 0$ is continuously differentiable, decreasing and convex on $(0, \infty)$ with $V_{n} \nearrow V$ as $n \uparrow \infty$. Hence, due to monotone integration, optimality of $\delta^{D}$ in (10) will follow by letting $n \uparrow \infty$ in $(33)$.

To prove this inequality, we first observe that, by definition and convexity of $V_{n}$,

$$
U(n)=V_{n}(0) \geq V_{n}\left(\delta^{D}\right)-V_{n}^{\prime}\left(\delta^{D}\right) \delta^{D} .
$$

By Assumption 2.1, $U(n)$ is $\mathbb{P} \otimes \mu$-integrable. Since $V_{n}, \delta^{D}$ and $-V_{n}^{\prime}$ are nonnegative, it thus follows that also

$$
-V_{n}^{\prime}\left(\delta^{D}\right) \delta^{D}=\left(C^{D} \wedge n\right) \delta^{D} \in \mathbf{L}^{1}(\mathbb{P} \otimes \mu)
$$


where the identity is due to the definition (32) of $C^{D}$.

Again by convexity of $V_{n}$, we have

$$
V_{n}(\delta)-V_{n}\left(\delta^{D}\right) \geq V_{n}^{\prime}\left(\delta^{D}\right)\left(\delta-\delta^{D}\right)=\left(C^{D} \wedge n\right) \delta^{D}-\left(C^{D} \wedge n\right) \delta .
$$

So to obtain (33), we have to show that the integral of the right side of (36) with respect to $\mathbb{P} \otimes \mu$ is nonnegative. To this end, note that

$$
\mathbb{E} \int_{0}^{\infty}\left(C^{D} \wedge n\right) \delta d \mu=\mathbb{E}\left\langle C^{D} \wedge n,{ }^{o} \int^{\infty} \delta d \mu\right\rangle \leq \mathbb{E}\left\langle C^{D} \wedge n, D\right\rangle,
$$

where the last estimate is immediate from $\delta \in \dot{\mathscr{D}}(D)$. When repeating this calculation for $\delta^{D}$ instead of $\delta$ this estimate turns into an identity because of $(13)$ and $\left\{d\left(C^{D} \wedge n\right)>0\right\} \subset\left\{d C^{D}>0\right\}$.

In conjunction with (35), it follows that indeed

$$
\mathbb{E} \int_{0}^{\infty}\left(C^{D} \wedge n\right) \delta d \mu \leq \mathbb{E} \int_{0}^{\infty}\left(C^{D} \wedge n\right) \delta^{D} d \mu<\infty
$$

This accomplishes our proof.

Lemma 5.2. Suppose Assumption 2.1 holds true. Then the conjugacy relation (15) holds. Moreover, if $\mathbb{U}(\hat{C})<\infty$, the infimum in (15) is attained for $D \in \mathscr{D}$ if and only if its envelope process with (12) and (13) is actually $\breve{D}=\int^{\infty} U^{\prime}(\hat{C}) d \mu$.

Proof. To prove " $\leq$ " in (15), take $D \in \mathscr{D}$ with $\mathbb{V}(D)<\infty$ and $\mathbb{E}\langle\hat{C}, D\rangle<$ $\infty$. By Lemma 5.1 there is $\delta^{D} \in \dot{\mathscr{D}}(D)$ such that $\mathbb{V}(D)=\mathbb{E} \int_{0}^{\infty} V\left(\delta^{D}\right) d \mu$. Then

$$
\mathbb{E} \int_{0}^{\infty} \hat{C} \delta^{D} d \mu=\mathbb{E}\left\langle\hat{C},{ }^{o} \int^{\infty} \delta^{D} d \mu\right\rangle \leq \mathbb{E}\langle\hat{C}, D\rangle<\infty .
$$

Thus we can integrate the inequality

$$
0 \leq U(\hat{C}) \leq V\left(\delta^{D}\right)+\hat{C} \delta^{D}
$$

with respect to $\mathbb{P} \otimes \mu$ to deduce that indeed

$$
\begin{aligned}
0 \leq \mathbb{U}(\hat{C})=\mathbb{E} \int_{0}^{\infty} U(\hat{C}) d \mu & \leq \mathbb{E} \int_{0}^{\infty} V\left(\delta^{D}\right) d \mu+\mathbb{E} \int_{0}^{\infty} \hat{C} \delta^{D} d \mu \\
& \leq \mathbb{V}(D)+\mathbb{E}\langle\hat{C}, D\rangle
\end{aligned}
$$


For " $\geq$ " in (15) we can assume $\mathbb{U}(\hat{C})=\mathbb{E} \int_{0}^{\infty} U(\hat{C}) d \mu<\infty$ without loss of generality. Let $\hat{\delta} \triangleq U^{\prime}(\hat{C})$ and note that because $U$ is concave in $c$ with $U(0)=0$ we have

$$
0 \leq \hat{C} \hat{\delta}=\hat{C} U^{\prime}(\hat{C}) \leq U(\hat{C}) \in \mathbf{L}^{1}(\mathbb{P} \otimes \mu) .
$$

Moreover, $\hat{D} \triangleq \int_{\text {. }}^{\infty} \hat{\delta} d \mu \in \mathscr{D}$ satisfies

$$
\mathbb{V}(\hat{D}) \leq \mathbb{E} \int_{0}^{\infty} V(\hat{\delta}) d \mu=\mathbb{E} \int_{0}^{\infty} U(\hat{C}) d \mu+\mathbb{E} \int_{0}^{\infty} \hat{C} \hat{\delta} d \mu<\infty .
$$

From Lemma 5.1 it now follows that in fact $\mathbb{V}(\hat{D})=\mathbb{E} \int_{0}^{\infty} V(\hat{\delta}) d \mu<\infty$. We thus can integrate the identity

$$
U(\hat{C})=V(\hat{\delta})-\hat{C} \hat{\delta}
$$

with respect to $\mathbb{P} \otimes \mu$ to obtain

$$
\mathbb{U}(\hat{C})=\mathbb{V}(\hat{D})-\mathbb{E} \int_{0}^{\infty} \hat{C} \hat{\delta} d \mu=\mathbb{V}(\hat{D})-\mathbb{E}\langle\hat{C}, \hat{D}\rangle .
$$

This gives " $\geq$ " in (15).

The preceding argument already establishes the "if"-part of the present lemma. For the "only if"-part assume that $D \in \mathscr{D}$ satisfies $\mathbb{U}(\hat{C})=\mathbb{V}(D)+$ $\mathbb{E}\langle\hat{C}, D\rangle<\infty$. Clearly, we have $\mathbb{V}(D)<\infty$ then. Thus, by Lemma 5.1, there is $\delta^{D} \in \dot{\mathscr{D}}(D)$ with $\mathbb{V}(D)=\mathbb{E} \int_{0}^{\infty} V\left(\delta^{D}\right) d \mu<\infty$. Moreover, the choice of $D$ entails $\mathbb{E} \int_{0}^{\infty} \hat{C} \delta^{D} d \mu \leq \mathbb{E}\langle\hat{C}, D\rangle<\infty$. Now, integrating

$$
U(\hat{C}) \leq V\left(\delta^{D}\right)+\hat{C} \delta^{D}
$$

we find

$$
\mathbb{U}(\hat{C}) \leq \mathbb{V}(D)+\mathbb{E}\left\langle\hat{C},{ }^{o} \int^{\infty} \delta^{D} d \mu\right\rangle \leq \mathbb{V}(D)+\mathbb{E}\langle\hat{C}, D\rangle=\mathbb{U}(\hat{C})<\infty
$$

So, equality must hold true in all the above estimates. It follows that equality holds $\mathbb{P} \otimes \mu$-almost everywhere in (38) which readily implies $\delta^{D}=U^{\prime}(\hat{C})$ $\mathbb{P} \otimes \mu$-almost everywhere, and, thus, ${ }^{o} \int^{\infty} U^{\prime}(\hat{C}) d \mu={ }^{o} \int^{\infty} \delta^{D} d \mu \leq{ }^{o} D$. Moreover, (39) then also yields $\mathbb{E}\left\langle\hat{C},{ }^{o} \int^{\infty}{ }^{\infty} U^{\prime}(\hat{C}) d \mu\right\rangle=\mathbb{E}\left\langle\hat{C},{ }^{o} D\right\rangle$, i.e., in fact ${ }^{o} \int^{\infty} U^{\prime}(\hat{C}) d \mu={ }^{o} D$ on $\{d \hat{C}>0\}$. By Lemma 5.1, this identifies $\int^{\infty} U^{\prime}(\hat{C}) d \mu$ as the envelope process $\breve{D}$ of $D$ with (12) and (13). This accomplishes our proof. 
Lemma 5.3. Suppose Assumption 2.1 holds. Then the conjugacy relation (16) holds. Moreover, if $\mathbb{V}(\hat{D})<\infty$, the supremum in (16) is attained exactly for $\hat{C} \triangleq C^{\hat{D}}$ where $C^{\hat{D}}$ is defined in Lemma 5.1.

Proof. Let us first apply Lemma 5.1 to obtain that there is $\hat{\delta} \triangleq \delta^{\hat{D}} \in \dot{\mathscr{D}}(\hat{D})$ with $\mathbb{V}(\hat{D})=\mathbb{E} \int_{0}^{\infty} V(\hat{\delta}) d \mu$.

To see that " $\geq$ " holds in (16), take $C \in \mathscr{C}$ with $\mathbb{U}(C)=\mathbb{E} \int_{0}^{\infty} U(C) d \mu<$ $\infty$. Without loss of generality we can assume $\mathbb{V}(\hat{D})<\infty$ and $\mathbb{E}\langle C, \hat{D}\rangle<\infty$. Then all terms in the inequality

$$
V(\hat{\delta}) \geq U(C)-C \hat{\delta}
$$

are $\mathbb{P} \otimes \mu$-integrable. Upon integration we get $\mathbb{V}(\hat{D}) \geq \mathbb{U}(C)-\mathbb{E}\left\langle C, \int^{\infty} \hat{\delta} d \mu\right\rangle$. This implies the desired estimate since ${ }^{o}\left(\int^{\infty} \hat{\delta} d \mu\right) \leq{ }^{o} \hat{D}$.

For the proof of " $\leq$ " in (16) consider $\hat{C} \triangleq-V^{\prime}(\hat{\delta}) \in \mathscr{C}$ where $\hat{\delta}$ is chosen as above. If $\mathbb{V}(\hat{D})=\infty$, we consider $C^{n} \triangleq \hat{C} \wedge n \in\{\mathbb{U}<\infty\}, n=1,2, \ldots$, in (16) to deduce

$\mathbb{U}\left(C^{n}\right)-\mathbb{E}\left\langle C^{n}, \hat{D}\right\rangle=\mathbb{E} \int_{0}^{\infty}\left(U(\hat{C} \wedge n)-(\hat{C} \wedge n) U^{\prime}(\hat{C} \wedge n) d \mu=\mathbb{E} \int_{0}^{\infty} V_{n}(\hat{\delta}) d \mu\right.$

where $V_{n}$ is as in (34). Since $V_{n} \nearrow V$, it follows by monotone integration that as $n \uparrow \infty$ the above expression converges to $\mathbb{E} \int_{0}^{\infty} V(\hat{\delta}) d \mu \geq \mathbb{V}(\hat{D})$ and we obtain " $\leq$ " in $(16)$ in case $\mathbb{V}(\hat{D})=\infty$.

For the remaining case where $\mathbb{E} \int_{0}^{\infty} V(\hat{\delta}) d \mu<\infty$, let us first show that $\mathbb{U}(\hat{C})=\mathbb{E} \int_{0}^{\infty} U(\hat{C}) d \mu<\infty$. Indeed, by Assumption 2.1 the asymptotic elasticity of $U$ is uniformly less than one in the sense that $c U^{\prime}(c)<\gamma U(c)$ for $c>C^{\gamma}$ where $\gamma \in[0,1)$. Thus, we have

$$
\mathbf{L}^{1}(\mathbb{P} \otimes \mu) \ni V(\hat{\delta})=U(\hat{C})-\hat{C} U^{\prime}(\hat{C}) \geq(1-\gamma) U(\hat{C}) \geq 0 \text { on }\left\{\hat{C}>C^{\gamma}\right\} .
$$

Since by assumption $\mathbb{E} \int_{0}^{\infty} U\left(C^{\gamma}\right) d \mu<\infty$, it thus follows that $U(\hat{C}) \in \mathbf{L}^{1}(\mathbb{P} \otimes$ $\mu)$, i.e., $\mathbb{U}(\hat{C})<\infty$.

Now, recalling the estimate (37), we deduce from $\mathbb{U}(\hat{C})<\infty$ that also $\mathbb{E}\langle\hat{C}, \hat{D}\rangle=\mathbb{E} \int_{0}^{\infty} \hat{C} \hat{\delta} d \mu<\infty$. The " $\leq$ "-claim now follows upon integration of $V(\hat{\delta})=U(\hat{C})-\hat{C} \hat{\delta}$ with respect to $\mathbb{P} \otimes \mu$. This also establishes the "if"part of our lemma. The "only if"-part follows immediately from this and the strict concavity of $\mathbb{U}$ on $\{\mathbb{U}<\infty\}$ which implies the uniqueness of the optimizer $\hat{C}$. 


\subsection{Proof of Theorem 3.2}

The proof of Theorem 3.2 is prepared by the following Lemmas 5.4-5.9.

Lemma 5.4. Under the assumptions of Theorem 3.2, we have

$$
\mathbf{v}(y)=\inf _{\delta \in \dot{\mathscr{D}}(y)} \mathbb{E} \int_{0}^{\infty} V(\delta) d \mu, \quad y>0,
$$

where

$$
\dot{\mathscr{D}}(y) \triangleq \bigcup_{D \in \mathscr{D}(y)} \dot{\mathscr{D}}(D)
$$

Moreover, for any $y>0$ with $\mathbf{v}(y)<\infty$, the infimum in (40) is attained at a unique $\delta^{y} \in \dot{\mathscr{D}}(y)$ for which, in addition, $\hat{C}^{y} \triangleq-V^{\prime}\left(\delta^{y}\right)$ is contained in $\mathscr{C}$. Finally, $\mathbf{v}$ is strictly convex on $\{\mathbf{v}<\infty\}$.

Proof. Identity (40) is immediate from (41) and Lemma 5.1.

Now assume $\mathbf{v}(y)<\infty$ and consider a minimizing sequence $\delta^{n} \in \dot{\mathscr{D}}(y)$ for (40). By Lemma A1.1 of Delbaen and Schachermayer [11] there is a sequence $\tilde{\delta}^{n}$ of convex combinations of $\delta^{n}, \delta^{n+1}, \ldots$ which converges $\mathbb{P} \otimes \mu$ almost everywhere to an optional $\delta^{y}$ taking values in $[0, \infty]$. In fact, $\delta^{y} \in$ $\dot{\mathscr{D}}(y)$ because $D^{y} \triangleq \int^{\infty} \delta^{y} d \mu \in \mathscr{D}(y)$, which holds since by Fatou's lemma

$$
\begin{aligned}
\mathbb{E}\left\langle C, D^{y}\right\rangle=\mathbb{E} \int_{0}^{\infty} C \delta^{y} d \mu & \leq \liminf _{n} \mathbb{E} \int_{0}^{\infty} C \tilde{\delta}^{n} d \mu \\
& =\liminf _{n} \mathbb{E}\left\langle C, \int^{\infty} \tilde{\delta}^{n} d \mu\right\rangle \leq x y
\end{aligned}
$$

for any $C \in \mathscr{C}(x), x>0$. Here the last inequality follows because $\tilde{\delta}^{n} \in \dot{\mathscr{D}}(y)$ by convexity of this set. Another application of Fatou's lemma reveals

$$
\mathbb{E} \int_{0}^{\infty} V\left(\delta^{y}\right) d \mu \leq \liminf _{n} \mathbb{E} \int_{0}^{\infty} V\left(\tilde{\delta}^{n}\right) d \mu \leq \liminf _{n} \mathbb{E} \int_{0}^{\infty} V\left(\delta^{n}\right) d \mu=\mathbf{v}(y)
$$

by convexity of $V$ and our choice of $\left(\delta^{n}\right)_{n=1,2, \ldots}$ as a minimizing sequence. This proves existence of a minimizer for (40). Uniqueness up to a $\mathbb{P} \otimes \mu$ null set follows from the strict convexity of $V$. In fact, applying Lemma 5.1 for $D \triangleq D^{y}$ reveals that $\delta^{y}$ has a predictable $\mathbb{P} \otimes \mu$-modification which is unique up to indistinguishability if we require, in addition, that $-V^{\prime}\left(\delta^{y}\right) \in \mathscr{C}$. Strict convexity of $\mathbf{v}$ on $\{\mathbf{v}<\infty\}$ now follows from strict convexity and strict monotonicity of $V$. 
Lemma 5.5. Under the assumptions of Theorem 3.2, the primal value function $\mathbf{u}$ of (19) is real-valued and conjugate to the dual value function $\mathbf{v}$ of (20) in the sense that (21) and (22) hold true.

Proof. The primal value function $\mathbf{u}$ is, by assumption, finite at some point $x>0$. Its concavity then yields that it is finite and, thus, continuous on all of $(0, \infty)$. Therefore, by classical duality results (cf., e.g., Theorem 12.2 in Rockafellar [23]), (21) follows from (22).

Let us first argue that " $\geq$ " holds in (22). So take $C \in \mathscr{C}(x)$ and $D \in$ $\mathscr{D}(y)$. Then $\mathbb{E}\langle C, D\rangle \leq x y$ and, by equation (15) of Theorem 3.1:

$$
\mathbb{U}(C)-x y \leq \mathbb{V}(D)+\mathbb{E}\langle C, D\rangle-x y \leq \mathbb{V}(D) .
$$

Taking the supremum over $C \in \mathscr{C}(x)$ and the infimum over $D \in \mathscr{D}(y)$ in this relation yields " $\geq$ " in $(22)$.

To obtain that also " $\leq$ " holds in (22), we shall employ the Minimax Theorem B.3 from the appendix with

- $\mathscr{A} \triangleq \mathscr{C}_{n} \triangleq\left\{C \in \mathscr{C}: C_{\infty} \leq n\right\}$ where $n \in\{1,2, \ldots\}$, a convexly compact subset of the space of left-continuous processes with bounded total variation endowed with the metric dist of (2); see Lemma B.2.

- $\mathscr{B} \triangleq \mathscr{D}(y)$ which can be viewed as a convex, closed subset of the space of right-continuous processes with $\mathbb{P}$-integrable total variation endowed with convergence with respect to the distance dist of (2) because $\mathbb{E} D_{0}=$ $\mathbb{E}\langle\mathbf{1}, D\rangle \leq y$ by assumption on $\mathscr{D}(y)=y \mathscr{D}(1)$; and with

- $\mathbb{H}(C, D) \triangleq \mathbb{U}(C)-\mathbb{E}\langle C, D\rangle$, which is convex (even linear) in $D \in \mathscr{B}=$ $\mathscr{D}(y)$ and concave and upper-semicontinuous in $C \in \mathscr{A}=\mathscr{C}_{n}$, because, with respect to the metric dist, $\mathbb{U}$ is continuous on $\mathscr{C}_{n}$ by dominated convergence and $\mathbb{E}\langle., D\rangle$ is lower-semicontinuous due to Lemma B.1.

We thus obtain that, for $n=1,2, \ldots$,

$$
\sup _{C \in \mathscr{C}_{n}} \inf _{D \in \mathscr{D}(y)}\{\mathbb{U}(C)-\mathbb{E}\langle C, D\rangle\}=\inf _{D \in \mathscr{D}(y)} \sup _{C \in \mathscr{C}_{n}}\{\mathbb{U}(C)-\mathbb{E}\langle C, D\rangle\}
$$

Let us next prove that, as $n \uparrow \infty$, the left side of (42) converges to $\sup _{0 \leq x<\infty}\{\mathbf{u}(x)-x y\}$. Clearly, with $\pi(C) \triangleq \sup _{D \in \mathscr{D}(1)} \mathbb{E}\langle C, D\rangle$ the limit of 
left side of (42) can be written as

$$
\begin{aligned}
\sup _{C \in \mathscr{C} \text { bounded }} \inf _{D \in \mathscr{D}(y)}\{\mathbb{U}(C)-\pi(C) y\} & =\sup _{0 \leq x<\infty} \sup _{C \in \mathscr{C}(x) \text { bounded }}\{\mathbb{U}(C)-x y\} \\
& =\sup _{0 \leq x<\infty}\{\mathbf{u}(x)-x y\}
\end{aligned}
$$

where the last identity holds because by monotone convergence $\mathbb{U}(C)=$ $\lim _{n} \mathbb{U}(C \wedge n), C \in \mathscr{C}$, so that the utility of any $C$ can be approximated by the utility of bounded controls.

Now the proof of the present lemma will be accomplished once we have shown that, as $n \uparrow \infty$, the right side of (42) tends to a limit which is not smaller than $\mathbf{v}(y)$. To this end, we first observe that

$$
\sup _{C \in \mathscr{C}_{n}}\{\mathbb{U}(C)-\mathbb{E}\langle C, D\rangle\}=\mathbb{E} \int_{0}^{\infty} V_{n}(-\dot{\breve{D}}) d \mu \text { for any } D \in \mathscr{D}(y)
$$

where $V_{n}$ is given by (34). Indeed, because ${ }^{o} D \geq{ }^{o} \breve{D}$, we have

$$
\mathbb{U}(C)-\mathbb{E}\langle C, D\rangle \leq \mathbb{U}(C)-\mathbb{E}\langle C, \breve{D}\rangle=\mathbb{E} \int_{0}^{\infty} U(C)-C(-\dot{\breve{D}}) d \mu
$$

where for $C \in \mathscr{C}_{n}$ the last integrand is not larger than $V_{n}(-\dot{\breve{D}})$. This proves " $\leq$ " in (43). For " $\geq$ " we just need to observe that $C \triangleq-V_{n}^{\prime}(-\dot{\vec{D}})=$ $-V^{\prime}(-\dot{\breve{D}}) \wedge n \in \mathscr{C}_{n}$ will give equality in both of the preceding estimates.

Due to (43), we can take $D^{n} \in \mathscr{D}(y)$ with $0 \leq \delta^{n} \triangleq-\dot{\breve{D}}^{n}$ such that $\mathbb{E} \int_{0}^{\infty} V_{n}\left(\delta^{n}\right) d \mu$ converges to the limit of the right side of (42) as $n \uparrow \infty$. By Lemma A1.1 in [11] there are $\tilde{\delta}^{n} \in \operatorname{conv}\left\{\delta^{n}, \delta^{n+1}, \ldots\right\}, n=1,2, \ldots$, which converge $\mathbb{P} \otimes \mu$-almost everywhere to some $\delta^{*} \geq 0$. Because all $\delta^{n}$ are contained in $\dot{\mathscr{D}}(y)$, so are, by convexity of this set, all the $\tilde{\delta}^{n}$. In fact, also $\delta^{*} \in \dot{\mathscr{D}}(y)$ because $D^{*} \triangleq \int_{\text {. }}^{\infty} \delta^{*} d \mu \in \mathscr{D}(y)$ as by Fatou's lemma

$$
\begin{aligned}
\mathbb{E}\left\langle C, D^{*}\right\rangle=\mathbb{E} \int_{0}^{\infty} C \delta^{*} d \mu & \leq \liminf _{n} \mathbb{E} \int_{0}^{\infty} C \tilde{\delta}^{n} d \mu \\
& =\liminf _{n} \mathbb{E}\left\langle C,{ }^{o} \int^{\infty} \tilde{\delta}^{n} d \mu\right\rangle \leq x y
\end{aligned}
$$

for any $C \in \mathscr{C}(x)$. 
It follows that for $N=1,2, \ldots$ :

$$
\begin{aligned}
\lim _{n} & \inf _{D \in \mathscr{D}(y)} \sup _{C \in \mathscr{C}_{n}}\{\mathbb{U}(C)-\mathbb{E}\langle C, D\rangle\}=\lim _{n} \mathbb{E} \int_{0}^{\infty} V_{n}\left(\delta^{n}\right) d \mu \\
& \geq \liminf _{n} \mathbb{E} \int_{0}^{\infty} V_{N}\left(\delta^{n}\right) d \mu \geq \liminf _{n} \mathbb{E} \int_{0}^{\infty} V_{N}\left(\tilde{\delta}^{n}\right) d \mu \geq \mathbb{E} \int_{0}^{\infty} V_{N}\left(\delta^{*}\right) d \mu \\
& \underset{N \uparrow \infty}{\longrightarrow} \mathbb{E} \int_{0}^{\infty} V\left(\delta^{*}\right) d \mu \geq \mathbf{v}(y)
\end{aligned}
$$

where the first estimate and the convergence follow from $V_{n} \geq V_{N} \nearrow V$ for $n \geq N \uparrow \infty$. The second estimate is due to the convexity of $V_{N}$ and the third is due to Fatou's lemma. The last estimate is immediate from Lemma 5.1 and $\delta^{*} \in \dot{\mathscr{D}}(y)$.

Lemma 5.6. Under the assumptions of Theorem 3.2, $\mathbf{v}$ of (20) is realvalued, strictly convex and strictly decreasing on $(0, \infty)$. Moreover, $\mathbf{u}$ of (19) is continuously differentiable on $(0, \infty)$ with $\mathbf{u}^{\prime}(\infty)=0$.

Proof. Let us first show that even

$$
\lim _{x \uparrow \infty} \mathbf{u}(x) / x=0 .
$$

Indeed, since $\mathbf{u}$ takes real values by Lemma 5.5, we can find, for $\varepsilon>0$ and $x>0$, a $C^{x, \varepsilon} \in \mathscr{C}(x)$ such that $\mathbf{u}(x) \leq \mathbb{U}\left(C^{x, \varepsilon}\right)+\varepsilon$. Then, by the equivalent formulation (5) of our asymptotic elasticity condition (3),

$$
U\left(C^{x, \varepsilon}\right) \leq x^{\gamma} U\left(C^{x, \varepsilon} / x\right) \text { on }\left\{C^{x, \varepsilon} \geq C^{\gamma}\right\} .
$$

Upon integration with respect to $\mathbb{P} \otimes \mu$ we thus obtain

$$
\begin{aligned}
\mathbf{u}(x) & \leq x^{\gamma} \mathbb{E} \int_{0}^{\infty} U\left(C^{x, \varepsilon} / x\right) d \mu+\mathbb{E} \int_{0}^{\infty} U\left(C^{\gamma}\right) d \mu+\varepsilon \\
& \leq x^{\gamma} \mathbf{u}(1)+\mathbb{E} \int_{0}^{\infty} U\left(C^{\gamma}\right) d \mu+\varepsilon,
\end{aligned}
$$

where we used that $C^{x, \varepsilon} / x \in \mathscr{C}(1)$. Since $\gamma \in[0,1)$, our claim (44) now follows upon division by $x \uparrow \infty$.

In conjunction with (44), the duality between $\mathbf{u}$ and $\mathbf{v}$ established in Lemma 5.5 yields that $\mathbf{v}(y)<\infty$ for $y>0$. By Lemma 5.1, $\mathbf{v}$ is thus strictly convex on $(0, \infty)$. This immediately implies that $\mathbf{v}$ is strictly decreasing, By classical convex duality results (e.g. Rockafellar [23]), strict convexity of $\mathbf{v}$ implies the differentiability of its conjugate $\mathbf{u}$ on $(0, \infty)$. By concavity and monotonicity, $0 \leq \mathbf{u}^{\prime}(x) \leq \mathbf{u}(x) / x$. So (44) also yields $\mathbf{u}^{\prime}(\infty)=0$. 
The following lemma is a minor adaptation of Lemmas 3.6 and 3.7 in Kramkov and Schachermayer [20]:

Lemma 5.7. Under the assumptions of Theorem 3.2, the minimizers $\delta^{y} \in$ $\dot{\mathscr{D}}(y)$ from Lemma 5.4 depend continuously on $y>0$ in the sense that the mapping

$$
(0, \infty) \ni y \mapsto\left(\delta^{y}, V\left(\delta^{y}\right),-V^{\prime}\left(\delta^{y}\right) \delta^{y}\right) \in \mathbf{L}^{0}(\mathbb{P} \otimes \mu) \times \mathbf{L}^{1}(\mathbb{P} \otimes \mu) \times \mathbf{L}^{1}(\mathbb{P} \otimes \mu)
$$

is continuous.

Proof. That the above mapping is indeed defined on all of $(0, \infty)$ is due to the finiteness of $\mathbf{v}$ on $(0, \infty)$ established in Lemma 5.6.

We first prove that $\delta^{y_{n}} \rightarrow \delta^{y}$ in $\mathbf{L}^{0}(\mathbb{P} \otimes \mu)$ for any $y_{n} \rightarrow y \in(0, \infty)$. If $\delta^{y_{n}}$ does not converge to $\delta^{y}$ in this sense then there is $\varepsilon>0$ such that

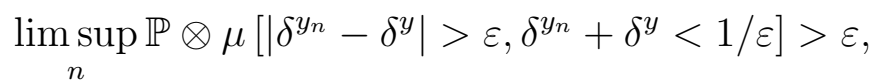

where we recall that $\left(\delta^{y_{n}}\right)_{n=1,2, \ldots}$ is bounded in $\mathbf{L}^{1}(\mathbb{P} \otimes \mu)$ because $\mathbb{E} \int_{0}^{\infty} \delta^{y_{n}} d \mu \leq$ $y_{n} \rightarrow y>0$ by definition of $\dot{\mathscr{D}}\left(y^{n}\right)$. Observe now that by strict convexity of $V, \delta^{n} \triangleq \frac{1}{2}\left(\delta^{y_{n}}+\delta^{y}\right)$ satisfies

$$
V\left(\delta^{n}\right) \leq \frac{1}{2}\left(V\left(\delta^{y_{n}}\right)+V\left(\delta^{y}\right)\right)
$$

and, for some sufficiently small $\eta>0$, also

$$
\limsup _{n} \mathbb{P} \otimes \mu\left[V\left(\delta^{n}\right) \leq \frac{1}{2}\left(V\left(\delta^{y_{n}}\right)+V\left(\delta^{y}\right)\right)-\eta\right]>\eta .
$$

Upon integration with respect to $\mathbb{P} \otimes \mu$ it follows that

$$
\begin{aligned}
\underset{n}{\limsup \mathbb{E} \int_{0}^{\infty} V\left(\delta^{n}\right) d \mu} & \leq \limsup _{n} \frac{1}{2} \mathbb{E} \int_{0}^{\infty}\left(V\left(\delta^{y_{n}}\right)+V\left(\delta^{y}\right)\right) d \mu-\eta^{2} \\
& =\limsup _{n} \frac{1}{2}\left(\mathbf{v}\left(y^{n}\right)+\mathbf{v}(y)\right)-\eta^{2}=\mathbf{v}(y)-\eta^{2}
\end{aligned}
$$

where the last identity is due to the continuity of the convex function v. On the other hand, by the scaling property and convexity of the sets $\mathscr{D}(y)=$ $y \mathscr{D}(1)$, we have $\delta^{n} \in \dot{\mathscr{D}}\left(y \vee y^{n}\right)$ and therefore, by Lemma 5.4,

$$
\mathbf{v}(y)=\lim _{n} \mathbf{v}\left(y \vee y^{n}\right) \leq \liminf _{n} \mathbb{E} \int_{0}^{\infty} V\left(\delta^{n}\right) d \mu .
$$


This clearly contradicts the preceding inequality and so we must have indeed that $\delta^{y_{n}} \rightarrow \delta^{y}$ in $\mathbf{L}^{0}(\mathbb{P} \otimes \mu)$.

Convergence of $V\left(\delta^{y_{n}}\right) \geq 0$ in $\mathbf{L}^{1}(\mathbb{P} \otimes \mu)$ now follows from convergence in $\mathbf{L}^{0}(\mathbb{P} \otimes \mu)$ and

$$
\mathbb{E} \int_{0}^{\infty} V\left(\delta^{y_{n}}\right) d \mu=\mathbf{v}\left(y^{n}\right) \underset{n \uparrow \infty}{\longrightarrow} \mathbf{v}(y)=\mathbb{E} \int_{0}^{\infty} V\left(\delta^{y}\right) d \mu .
$$

Moreover, $\mathbf{L}^{1}(\mathbb{P} \otimes \mu)$-convergence of $\left(-V^{\prime}\left(\delta^{y_{n}}\right) \delta^{y_{n}}\right)_{n=1,2, \ldots}$ will follow once we have established the uniform $\mathbb{P} \otimes \mu$-integrability of this sequence. Our uniform asymptotic elasticity condition (7) gives

$$
(1-\gamma)\left(-V^{\prime}\left(\delta^{y_{n}}\right)\right) \delta^{y_{n}} \leq \gamma V\left(\delta^{y_{n}}\right) \text { on }\left\{\delta^{y_{n}}<D^{\gamma}\right\}
$$

where $\gamma \in(0,1)$ and where $D^{\gamma} \triangleq U^{\prime}\left(C^{\gamma}\right)$. Moreover, we have, with $C^{n} \triangleq$ $-V^{\prime}\left(\delta^{y_{n}}\right)$, that

$$
0 \leq\left(-V^{\prime}\left(\delta^{y_{n}}\right)\right) \delta^{y_{n}}=C^{n} U^{\prime}\left(C^{n}\right) \leq U\left(C^{n}\right) \leq U\left(C^{\gamma}\right)
$$

on $\left\{\delta^{y_{n}} \geq D^{\gamma}\right\}=\left\{C^{n} \leq C^{\gamma}\right\}$. In conjunction with the already established $\mathbf{L}^{1}(\mathbb{P} \otimes \mu)$-convergence of $\left(V\left(\delta^{y_{n}}\right)\right)_{n=1,2, \ldots}$ and our assumption that $U\left(C^{\gamma}\right)$ is $\mathbb{P} \otimes \mu$-integrable, the combination of the estimates (45) and (46) yields the desired uniform integrability.

We now can use a variant of the argument in Lemma 3.8 of Kramkov and Schachermayer [20] to deduce:

Lemma 5.8. Under the assumptions of Theorem 3.2, the dual value function $\mathbf{v}$ is continuously differentiable on $(0, \infty)$ with

$$
\mathbf{v}^{\prime}(y) y=\mathbb{E} \int_{0}^{\infty} V^{\prime}\left(\delta^{y}\right) \delta^{y} d \mu, \quad y>0
$$

and $\mathbf{v}^{\prime}(0)=-\infty, \mathbf{v}^{\prime}(\infty)=0$. Moreover, $\mathbf{u}$ is strictly increasing and strictly concave on $(0, \infty)$ with $\mathbf{u}^{\prime}(0)=\infty$.

Proof. We first observe that for $y>0$ and $\lambda_{n} \downarrow 1$,

$$
\left(-V^{\prime}\left(\delta^{\lambda_{n} y} / \lambda_{n}\right) \delta^{\lambda_{n} y}\right)_{n=1,2, \ldots} \text { is uniformly } \mathbb{P} \otimes \mu \text {-integbrale. }
$$


Indeed, by employing successively our dual asymptotic elasticity estimates (7) and (8) and also the monotonicity of $V$, we get

$$
\begin{aligned}
0 \leq-V^{\prime}\left(\delta^{\lambda_{n} y} / \lambda_{n}\right) \delta^{\lambda_{n} y} & \leq \frac{\gamma}{1-\gamma} V\left(\delta^{\lambda_{n} y} / \lambda_{n}\right) \\
& \leq \frac{\gamma}{1-\gamma} V\left(\left(\delta^{\lambda_{n} y} \wedge D^{\gamma}\right) / \lambda_{n}\right) \\
& \leq \frac{\gamma}{1-\gamma}\left(\frac{1}{\lambda_{n}}\right)^{\frac{\gamma}{1-\gamma}} V\left(\delta^{\lambda_{n} y} \wedge D^{\gamma}\right) \\
& =\frac{\gamma}{1-\gamma}\left(\frac{1}{\lambda_{n}}\right)^{\frac{\gamma}{1-\gamma}}\left(V\left(\delta^{\lambda_{n} y}\right) \vee V\left(D^{\gamma}\right)\right)
\end{aligned}
$$

on $\left\{\delta^{\lambda_{n} y} / \lambda_{n} \leq D^{\gamma}\right\}$. With $C^{\lambda_{n}} \triangleq-V^{\prime}\left(\delta^{\lambda_{n} y} / \lambda_{n}\right)$ the complement of this set is $\left\{\delta^{\lambda_{n} y} / \lambda_{n}>D^{\gamma}\right\}=\left\{C_{n}^{\lambda}<C^{\gamma}\right\}$ and so, on this set,

$$
0 \leq-V^{\prime}\left(\delta^{\lambda_{n} y} / \lambda_{n}\right) \delta^{\lambda_{n} y}=U^{\prime}\left(C^{\lambda_{n}}\right) C_{n}^{\lambda} \lambda_{n} \leq U\left(C^{\lambda_{n}}\right) \lambda_{n} \leq U\left(C^{\gamma}\right) \lambda_{n}
$$

Hence, to obtain our claim (48) it suffices to observe that on either set we find an upper bound which is uniformly integrable. This is clear for $\left(U\left(C^{\gamma}\right) \lambda_{n}\right)_{n=1,2, \ldots}$. On the other hand, Lemma 5.7 yields in particular the uniform $\mathbb{P} \otimes \mu$-integrability of $\left(V\left(\delta^{\lambda_{n} y}\right)\right)_{n=1,2, \ldots}$ and finally $V\left(D^{\gamma}\right) \leq U\left(C^{\gamma}\right) \in$ $\mathbf{L}^{1}(\mathbb{P} \otimes \mu)$.

We now can argue exactly as in Lemma 3.8 of Kramkov and Schachermayer [20] and pass to the limit $\lambda \downarrow 1$ in

$$
\frac{\mathbf{v}(\lambda y)-\mathbf{v}(y)}{\lambda-1} \leq \mathbb{E} \int_{0}^{\infty} \frac{V\left(\lambda \delta^{y}\right)-V\left(\delta^{y}\right)}{\lambda-1} d \mu
$$

by monotone convergence and, by uniform integrability, also in

$$
\begin{aligned}
\frac{\mathbf{v}(\lambda y)-\mathbf{v}(y)}{\lambda-1} & \geq \mathbb{E} \int_{0}^{\infty} \frac{V\left(\delta^{\lambda y}\right)-V\left(\delta^{\lambda y} / \lambda\right)}{\lambda-1} d \mu \\
& \geq \mathbb{E} \int_{0}^{\infty} V^{\prime}\left(\delta^{\lambda y} / \lambda\right) \delta^{\lambda y} \frac{1-1 / \lambda}{\lambda-1} d \mu
\end{aligned}
$$

to see, respectively, that $(\mathbf{v}(\lambda y)-\mathbf{v}(y)) /(\lambda-1)$ has a lim sup not larger and a lim inf not smaller than the right side of (47). The continuity of this expression established in Lemma 5.7 in conjunction with the convexity of $\mathbf{v}$ then implies our claim. 
The strict monotonicity of $\mathbf{u}$ now follows from its strict concavity on $(0, \infty)$ which, in turn, is equivalent to the differentiability of its convex conjugate $\mathbf{v}$ on $(0, \infty)$ by classical duality results; see, e.g., Theorem 26.3 in Rockafellar [23]. These same results also yield the equivalence of $\mathbf{v}^{\prime}(\infty)=0$ and $\mathbf{u}^{\prime}(0)=\infty$, and the first of these relations is immediate from the fact that $\mathbf{v}$ is strictly convex and decreasing and bounded from below (by 0). Similarly, classical duality yields the equivalence of $\mathbf{v}^{\prime}(0)=-\infty$ and $\mathbf{u}^{\prime}(\infty)=0$, where the last relation was already established in Lemma 5.6.

Lemma 5.9. Under the assumptions of Theorem 3.2, $C^{x} \triangleq-V^{\prime}\left(\delta^{y}\right)$ with $y=\mathbf{u}^{\prime}(x)$ and $\delta^{y}$ as in Lemma 5.4 is the unique control in $\mathscr{C}(x)$ that attains $\mathbf{u}(x)=\mathbb{U}\left(C^{x}\right)$ for $x>0$.

Proof. Uniqueness of a maximizer $C^{x}$ for $\mathbf{u}(x)$ is immediate from the strict concavity of $U$. Define $D^{y} \triangleq \int^{\infty} \delta^{y} d \mu \in \mathscr{D}(y)$ and observe that due to (15) in Theorem 3.1 we have for any $C \in \mathscr{C}(x)$ :

$$
\mathbb{U}(C) \leq \mathbb{V}\left(D^{y}\right)+\mathbb{E}\left\langle C, D^{y}\right\rangle \leq \mathbb{V}\left(D^{y}\right)+x y
$$

By item 3. of Theorem 3.1 and by (47), we have equalities in both of these estimates when we consider $C \triangleq C^{x}=-V^{\prime}\left(\delta^{y}\right)$. It thus suffices to prove that $C^{x} \in \mathscr{C}(x)$, i.e., that

$$
\mathbb{E}\left\langle C^{x}, D\right\rangle \leq x y \text { for any } D \in \mathscr{D}(y) .
$$

For this, we first note that, for any such $D$, we have

$$
D^{\varepsilon} \triangleq \varepsilon D+(1-\varepsilon) D^{y} \in \mathscr{D}(y) \text { with } \mathbb{V}\left(D^{\varepsilon}\right)<\infty, \quad 0<\varepsilon<1 .
$$

Indeed, by monotonicity of $\mathbb{V}$,

$$
\mathbb{V}\left(D^{\varepsilon}\right) \leq \mathbb{V}\left((1-\varepsilon) D^{y}\right) \leq \mathbb{E} \int_{0}^{\infty} V\left((1-\varepsilon) \delta^{y}\right) d \mu
$$

so that for $(50)$ it suffices to argue that $V\left((1-\varepsilon) \delta^{y}\right)$ is $\mathbb{P} \otimes \mu$-integrable. To this end, we use the asymptotic elasticity condition (8) which, in conjunction with the monotonicity of $V$, gives

$$
\begin{aligned}
V\left((1-\varepsilon) \delta^{y}\right) & \leq V\left((1-\varepsilon)\left(\delta^{y} \wedge D^{\gamma}\right)\right) \\
& \leq(1-\varepsilon)^{\frac{\gamma}{1-\gamma}} V\left(\delta^{y} \wedge D^{\gamma}\right)=(1-\varepsilon)^{\frac{\gamma}{1-\gamma}} V\left(\delta^{y}\right) \vee V\left(D^{\gamma}\right)
\end{aligned}
$$


Hence, the integrability claim of $(50)$ follows since $V\left(\delta^{y}\right) \in \mathbf{L}^{1}(\mathbb{P} \otimes \mu)$ by choice of $\delta^{y}$ and $V\left(D^{\gamma}\right)=U\left(C^{\gamma}\right)-C^{\gamma} U^{\prime}\left(C^{\gamma}\right) \in \mathbf{L}^{1}(\mathbb{P} \otimes \mu)$ by assumption on $C^{\gamma}$.

Because of (50), we can apply Theorem 3.1 part 3. to deduce that there is a unique $C^{\varepsilon} \in \mathscr{C}$ such that $\mathbb{V}\left(D^{\varepsilon}\right)=\mathbb{U}\left(C^{\varepsilon}\right)-\mathbb{E}\left\langle C^{\varepsilon}, D^{\varepsilon}\right\rangle$. Moreover, (16) of Theorem 3.1 gives $\mathbb{V}\left(D^{y}\right) \geq \mathbb{U}\left(C^{\varepsilon}\right)-\mathbb{E}\left\langle C^{\varepsilon}, D^{y}\right\rangle$. Recalling the minimality of $\mathbb{V}\left(D^{y}\right)$ we thus obtain

$$
0 \leq \mathbb{V}\left(D^{\varepsilon}\right)-\mathbb{V}\left(D^{y}\right) \leq \mathbb{E}\left\langle C^{\varepsilon}, D^{y}-D^{\varepsilon}\right\rangle=\varepsilon \mathbb{E}\left\langle C^{\varepsilon}, D^{y}-D\right\rangle .
$$

Therefore,

$$
0 \leq \mathbb{E}\left\langle C^{\varepsilon}, D\right\rangle \leq \mathbb{E}\left\langle C^{\varepsilon}, D^{y}\right\rangle \leq \frac{1}{1-\varepsilon} \mathbb{E}\left\langle C^{\varepsilon}, D^{\varepsilon}\right\rangle
$$

where the last estimate is immediate from $D^{y} \leq D^{\varepsilon} /(1-\varepsilon)$. Hence, (49) will follow from letting $\varepsilon \downarrow 0$ in (51) once we have established that

$$
\mathbb{E}\left\langle C^{x}, D\right\rangle \leq \liminf _{\varepsilon \downarrow 0} \mathbb{E}\left\langle C^{\varepsilon}, D\right\rangle
$$

and

$$
\lim _{\varepsilon \downarrow 0} \mathbb{E}\left\langle C^{\varepsilon}, D^{\varepsilon}\right\rangle=x y
$$

To obtain this it suffices to consider a sequence $\varepsilon_{n} \downarrow 0$ and prove

$$
\operatorname{dist}\left(C^{\varepsilon_{n}}, C^{x}\right) \rightarrow 0 \text { as } n \uparrow \infty
$$

for the distance dist of (2) and

$$
C^{\varepsilon_{n}} U^{\prime}\left(C^{\varepsilon_{n}}\right) \underset{n \uparrow \infty}{\longrightarrow} C^{x} U^{\prime}\left(C^{x}\right)=C^{x} \delta^{y} \text { in } \mathbf{L}^{1}(\mathbb{P} \otimes \mu) .
$$

Indeed, the lower semi-continuity of the bracket $\mathbb{E}\langle., D\rangle$ with respect to convergence in dist (Lemma B.1) then yields (52). Similarly (53) follows because (55) yields

$$
\mathbb{E}\left\langle C^{x}, D^{y}\right\rangle=\mathbb{E} \int_{0}^{\infty} C^{x} \delta^{y} d \mu=\lim _{n} \mathbb{E} \int_{0}^{\infty} C^{\varepsilon_{n}} U^{\prime}\left(C^{\varepsilon_{n}}\right) d \mu=\lim _{n} \mathbb{E}\left\langle C^{\varepsilon_{n}}, D^{\varepsilon_{n}}\right\rangle
$$

and because (47) yields that $\mathbb{E}\left\langle C^{x}, D^{y}\right\rangle=x y$ by choice of $x$ and $y$. 
For (54) we will in fact prove that $\delta^{\varepsilon_{n}} \triangleq U^{\prime}\left(C^{\varepsilon_{n}}\right) \rightarrow \delta^{y}=U^{\prime}\left(C^{x}\right)$ in $\mathbf{L}^{0}(\mathbb{P} \otimes \mu)$. If this convergence fails there is $\varepsilon>0$ such that

$$
\limsup _{n} \mathbb{P} \otimes \mu\left[\left|\delta^{\varepsilon_{n}}-\delta^{y}\right|>\varepsilon\right]>\varepsilon
$$

Observe now that by strict convexity of $V, \delta^{n} \triangleq \frac{1}{2}\left(\delta^{\varepsilon_{n}}+\delta^{y}\right) \in \dot{\mathscr{D}}(y)$ satisfies

$$
V\left(\delta^{n}\right) \leq \frac{1}{2}\left(V\left(\delta^{\varepsilon_{n}}\right)+V\left(\delta^{y}\right)\right)
$$

and, for some sufficiently small $\eta>0$, also

$$
\limsup _{n} \mathbb{P} \otimes \mu\left[V\left(\delta^{n}\right) \leq \frac{1}{2}\left(V\left(\delta^{\varepsilon_{n}}\right)+V\left(\delta^{y}\right)\right)-\eta\right]>\eta
$$

Upon integration with respect to $\mathbb{P} \otimes \mu$ we obtain the contradiction

$$
\begin{aligned}
\mathbf{v}(y) & \leq \limsup _{n} \mathbb{E} \int_{0}^{\infty} V\left(\delta^{n}\right) d \mu \\
& \leq \limsup _{n} \frac{1}{2} \mathbb{E} \int_{0}^{\infty}\left(V\left(\delta^{\varepsilon_{n}}\right)+V\left(\delta^{y}\right)\right) d \mu-\eta^{2} \\
& =\limsup _{n} \frac{1}{2}\left(\mathbb{V}\left(\varepsilon_{n} D+\left(1-\varepsilon_{n}\right) D^{y}\right)+\mathbb{V}\left(D^{y}\right)\right)-\eta^{2} \\
& \leq \mathbb{V}\left(D^{y}\right)-\eta^{2}<\mathbf{v}(y)
\end{aligned}
$$

where the last but one estimate is due to the upper-semicontinuity of the convex function $[0,1] \ni \varepsilon \mapsto \mathbb{V}\left(\varepsilon D+(1-\varepsilon) D^{y}\right)$ at the boundary point 0 . Hence, we must have indeed that $\delta^{y_{n}} \rightarrow \delta^{y}$ in $\mathbf{L}^{0}(\mathbb{P} \otimes \mu)$.

In light of (54), (55) will follow once we have established the uniform integrability of $\left(C^{\varepsilon_{n}} U^{\prime}\left(C^{\varepsilon_{n}}\right)\right)_{n=1,2, \ldots}$. On $\left\{C^{\varepsilon_{n}} \leq C^{\gamma}\right\}$, we have $C^{\varepsilon_{n}} U^{\prime}\left(C^{\varepsilon_{n}}\right) \leq$ $U\left(C^{\varepsilon_{n}}\right) \leq U\left(C^{\gamma}\right) \in \mathbf{L}^{1}(\mathbb{P} \otimes \mu)$ by assumption on $C^{\gamma}$. On $\left\{C^{\varepsilon_{n}}>C^{\gamma}\right\}=$ $\left\{\delta^{\varepsilon_{n}}<D^{\gamma}\right.$, we have $C^{\varepsilon_{n}} U^{\prime}\left(C^{\varepsilon_{n}}\right)=-\delta^{\varepsilon_{n}} V^{\prime}\left(\delta^{\varepsilon_{n}}\right) \leq \frac{\gamma}{1-\gamma} V\left(\delta^{\varepsilon_{n}}\right)$ by our asymptotic elasticity assumption. So it suffices to prove the $\mathbf{L}^{1}(\mathbb{P} \otimes \mu)$-convergence of $\left(V\left(\delta^{\varepsilon_{n}}\right)\right)_{n=1,2, \ldots}$. Because this sequence is convergent in $\mathbf{L}^{0}(\mathbb{P} \otimes \mu)$ and nonnegative, this amounts to showing that $\lim _{n} \mathbb{E} \int_{0}^{\infty} V\left(\delta^{\varepsilon_{n}}\right) d \mu=\mathbb{E} \int_{0}^{\infty} V\left(\delta^{y}\right) d \mu$.

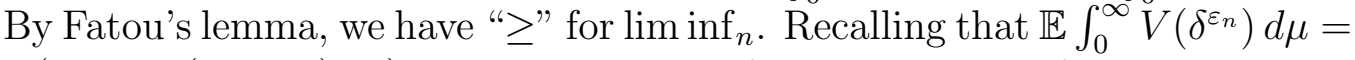
$\mathbb{V}\left(\varepsilon_{n} D+\left(1-\varepsilon_{n}\right) D^{y}\right)$, we deduce " $\leq$ " for the limsup $\operatorname{sum}_{n}$ from the uppersemicontinuity of the convex function $[0,1] \ni \varepsilon \mapsto \mathbb{V}\left(\varepsilon D+(1-\varepsilon) D^{y}\right)$ at the boundary point 0 . 
We now can finally give the

Proof of Theorem 3.2. For item 1. we note that $\mathbf{u}$ and $\mathbf{v}$ are realvalued by Lemmas 5.5 and 5.6, respectively. Their duality is established in Lemma 5.5 and their differentiability is contained in Lemmas 5.6 and 5.8, respectively. The Inada conditions (23) can be collected from Lemmas 5.6 and 5.8. The conjugacy relations between optimizers for $\mathbf{u}$ and $\mathbf{v}$ follow from the duality of $\mathbf{u}$ and $\mathbf{v}$. Strict concavity of $\mathbf{u}$ is similarly a consequence of the differentiability of $\mathbf{v}$; see Theorem 26.3 in Rockafellar [23].

Item 3. is just a dual formulation of item 2. For $y>0$, Lemma 5.4 yields $\delta^{y} \in \dot{\mathscr{D}}(y)$ with $\mathbf{v}(y)=\mathbb{E} \int_{0}^{\infty} V\left(\delta^{y}\right) d \mu$. This readily implies that $\breve{D}^{y} \triangleq$ $\int^{\infty} \delta^{y} d \mu$ is contained in $\mathscr{D}(y)$ and attains the infimum in (20). Lemma 5.9 shows that $C^{x} \triangleq-V^{\prime}\left(\delta^{y}\right)$ attains $\mathbf{u}(x)=\mathbb{U}\left(C^{x}\right)$. Let now $\tilde{D} \in \mathscr{D}(y)$ also attain $\mathbf{v}(y)=\mathbb{V}(\tilde{D})$. We then have

$$
\mathbb{U}\left(C^{x}\right)=\mathbf{u}(x)=\mathbf{v}(y)+x y \geq \mathbb{V}(\tilde{D})+\mathbb{E}\left\langle C^{x}, \tilde{D}\right\rangle,
$$

i.e. $\tilde{D}$ attains the infimum (15) for $\hat{C} \triangleq C^{x}$. It thus follows by item 2 . of Theorem 3.1 that $\tilde{D}$ has an envelope process whose density coincides with $-U^{\prime}\left(C^{x}\right)=\delta^{y}$. Hence, the envelope process of all the minimizers of (20) is the same process $\breve{D}^{y}$. This accomplishes our proof.

\section{A Some stochastic envelope processes}

The existence of envelope processes $\breve{D}$ with (12) and (13) for $D \in \mathscr{D}$ is key for our approach. We show below how to obtain such an envelope from a result in Bank and El Karoui [3]. Uniqueness is established by an optimal stopping argument which we adopt from Bank and Föllmer [4].

Lemma A.1. Under Assumption 2.1, any $D \in \mathscr{D}$ has a unique (up to indistinguishability) envelope process $\breve{D}$ of the form

$$
\breve{D}_{t}=\int_{t}^{\infty} U^{\prime}\left(C^{\breve{D}}\right) d \mu, t \geq 0, \text { for some } C^{\breve{D}} \in \mathscr{C}
$$

such that $\mathbb{P}$-a.s.

$$
{ }^{o} \breve{D}_{t} \leq{ }^{o} D_{t} \text { for any } t \geq 0 \text {, with "=" if } d C_{t}^{\breve{D}}>0 \text {. }
$$


Proof. For existence we will employ Theorem 2 of [3], which, however, we cannot directly apply with $X \triangleq{ }^{o} D$ because ${ }^{o} D$ may not be of class (D). So let $S^{n} \triangleq \inf \left\{t \geq 0: D_{t} \leq n\right\}$ and put $X^{n} \triangleq{ }^{o} D_{. \vee S^{n}}$ for $n=1,2, \ldots$ Then, because $D$ is right-continuous and non-increasing, $X^{n}$ is even bounded and clearly lower-semicontinuous in expectation with $X_{\infty}^{n}=0$. Moreover, let

$$
f_{t}(\omega, l) \triangleq \begin{cases}U_{t}^{\prime}(\omega,-1 / l), & l<0 \\ -l, & l \geq 0\end{cases}
$$

Then, by the properties of $U$ :

- $l \mapsto f_{t}(\omega, l)$ is a continuous function, strictly decreasing from $+\infty$ to $-\infty$ in $l \in(-\infty, \infty)$ for any $(\omega, t) \in \Omega \times[0, \infty)$, and

- $(\omega, t) \mapsto f_{t}(\omega, l)$ is a predictable $\mathbb{P} \otimes \mu$-integrable process on $\Omega \times[0, \infty)$ for any $l \in(-\infty, \infty)$.

So, by Theorem 2 of [3] and their Remark 2.1, there exists an optional process $L^{n}$ such that

$$
X_{S}^{n}=\mathbb{E}\left[\int_{S}^{\infty} f_{t}\left(\sup _{v \in[S, t)} L_{v}^{n}\right) d \mu_{t} \mid \mathscr{F}_{S}\right]
$$

for any stopping time $S \geq 0$. Clearly, we may assume that $L^{n}=L^{n+1}$ on $\left(S^{n}, \infty\right)$. So

$$
L_{t} \triangleq \begin{cases}L_{t}^{n}, & t \in\left(S^{n}, \infty\right), n=1,2, \ldots, \\ -\infty, & t \in\left[0, S^{\infty}\right],\end{cases}
$$

where $S^{\infty}=\inf _{n} S^{n}=\inf \left\{t \geq 0:{ }^{o} D_{t}<\infty\right\}$, consistently defines an optional process $L$ such that

$$
{ }^{o} D_{S}=\mathbb{E}\left[\int_{S}^{\infty} f_{t}\left(\sup _{v \in[S, t)} L_{v}\right) d \mu_{t} \mid \mathscr{F}_{S}\right]
$$

for any stopping time $S \geq 0$.

Let us next argue that $L \leq 0$ up to indistinguishability. Otherwise there exists, by Meyer's optional section theorem, a stopping time $S$ such that $L_{S}>0$ on $\{S<\infty\}$ where the latter set has positive probability. But then we obtain, by definition of $f$,

$$
0 \leq{ }^{o} D_{S}=\mathbb{E}\left[\int_{S}^{\infty} f_{t}\left(\sup _{v \in[S, t)} L_{v}\right) d \mu_{t} \mid \mathscr{F}_{S}\right] \leq-L_{S} \mathbb{E}\left[\mu([S, \infty)) \mid \mathscr{F}_{S}\right]<0
$$


on $\{S<\infty\}$, a contradiction.

It follows that

$$
C_{t}^{\breve{D}} \triangleq \begin{cases}0, & t \in\left[0, S^{\infty}\right], \\ -1 / \sup _{s \in[0, t)} L_{s}, & t \in\left(S^{\infty}, \infty\right],\end{cases}
$$

and $\breve{D} \triangleq \int_{.}^{\infty} U^{\prime}\left(C^{\breve{D}}\right) d \mu$ yield processes contained in $\mathscr{C}$ and $\mathscr{D}$, respectively, with the desired properties (56) and (57).

Let us now prove uniqueness of such a $\breve{D}$ and take an arbitrary $\tilde{C} \in \mathscr{C}$ such that $\tilde{D}=\int^{\infty} U^{\prime}(\tilde{C}) d \mu \in \mathscr{D}$ satisfies ${ }^{\circ} \tilde{D} \leq{ }^{o} D$, with "=" on $\{d \tilde{C}>0\}$. We will show that, for any $l>0, \tilde{S}^{l} \triangleq \inf \left\{t \geq 0: \tilde{C}_{t}>l\right\}$ is the largest stopping time minimizing $\mathbb{E}\left[D_{S}-\int_{S}^{\infty} U^{\prime}(l) d \mu\right]$ over all stopping times $S \geq$ 0 . As a result, the level passage times for $\tilde{C}$ are uniquely determined and, thus, have to coincide with those of $C^{\breve{D}}$, proving that $\tilde{C}=C^{\breve{D}}$, i.e., $\tilde{D}=\breve{D}$ up to indistinguishability.

For our optimal stopping claim, we first note that $0 \leq U^{\prime}(l) \leq U(l) / l \in$ $\mathbf{L}^{1}(\mathbb{P} \otimes \mu)$ for $l>0$ and so the above optimal stopping problem is well-defined. Now take a stopping time $S \geq 0$ and observe that

$$
\begin{aligned}
\mathbb{E}\left[D_{S}-\int_{S}^{\infty} U^{\prime}(l) d \mu\right] & \geq \mathbb{E}\left[\int_{S}^{\infty}\left\{U^{\prime}(\tilde{C})-U^{\prime}(l)\right\} d \mu\right] \\
& \geq \mathbb{E}\left[\int_{\tilde{S}^{l}}^{\infty}\left\{U^{\prime}(\tilde{C})-U^{\prime}(l)\right\} d \mu\right]
\end{aligned}
$$

where the first inequality is due to ${ }^{\circ} D \geq{ }^{o} \tilde{D}$ and the second follows by definition of $\tilde{S}^{l}$ and monotonicity of $c \mapsto U^{\prime}(c)$. For $S=\tilde{S}^{l}$ the properties of $\tilde{C}$ ensure that we have equality everywhere in the above estimates and so $\tilde{S}^{l}$ solves our optimal stopping problem. Moreover, the strict monotonicity of $c \mapsto U^{\prime}(c)$ ensures that any stopping time $S>\tilde{S}^{l}$ will yield a strict inequality in the last estimate above and so $\tilde{S}^{l}$ is in fact the largest solution to the stopping problem, as remained to be shown.

\section{B Convex compactness and a minimax theo- rem}

In this section we first collect a few properties of subsets of $\mathscr{C}$ related to the pairing (1). In particular, we investigate the induced notion of convex 
compactness. For the sake of completeness, we also provide a version of the well-known minimax theorem which is adapted to this generalized notion of compactness.

Lemma B.1. The pairing $(C, D) \mapsto \mathbb{E}\langle C, D\rangle$ is lower-semicontinuous with respect to convergence in the metric dist of (2) in each of its factors.

Proof. The argument for lower-semicontinuity with respect to $D$ being similar, let us show lower-semicontinuity with respect to $C \in \mathscr{C}$ for fixed $D \in \mathscr{D}$.

By Fatou's lemma we have

$$
\liminf _{n} \mathbb{E}\left\langle C^{n}, D\right\rangle=\liminf _{n} \mathbb{E} \int_{(0, \infty]} C^{n}|d D| \geq \mathbb{E} \int_{(0, \infty]} \liminf _{n} C^{n}|d D| .
$$

Now $\operatorname{dist}\left(C^{n}, C\right) \rightarrow 0$ implies $\lim _{n} C^{n}=C$ on $\{\Delta C=0\}$ whose countable complement is a $|d D|$-null set $\mathbb{P}$-almost surely if $D$ is continuous.

An arbitrary $D \in \mathscr{D}$ is right-continuous and non-increasing. We thus can find continuous, real-valued $D^{m} \in \mathscr{D}$ with $D^{m} \nearrow D$ pointwise as $m \uparrow \infty$. So, since our claim holds for these continuous $D^{m}$, we can conclude

$$
\liminf _{n} \mathbb{E}\left\langle C^{n}, D\right\rangle \geq \liminf _{n} \mathbb{E}\left\langle C^{n}, D^{m}\right\rangle \geq \mathbb{E}\left\langle C, D^{m}\right\rangle=\mathbb{E} \int_{[0, \infty)} D^{m} d C
$$

for $m=1,2, \ldots$ The claim for $D$ then follows by monotone integration as we let $m \uparrow \infty$ in the last term of the above inequality.

Recall from Žitković [26], Definition 2.1, that a subset of a topological vector space is convexly compact if it satisfies the finite intersection property for closed and convex subsets. Equivalently, a closed and convex subset of a topological vector space is convexly compact if and only if for every net in this set there exists a convergent subnet of convex combinations (cf. Proposition 2.4 in [26]).

We use convex compact sets in the Minimax Theorem B.3 below. The connection with our duality framework of Lemma 5.5 is made possible by the following result.

Lemma B.2. Let $\mathscr{A}$ be a convex subset of the consumption space $\mathscr{C}$ that is closed in the topology generated by the metric dist of (2). Then $\mathscr{A}$ is convexly compact if and only if the set of random variables $\left\{C_{\infty}: C \in \mathscr{E}\right\}$ is bounded in probability. 
In particular, for any $c \in[0, \infty),\left\{C \in \mathscr{C}: C_{\infty} \leq c\right\}$ is a convexly compact subset of the space of left-continuous processes with bounded total variation endowed with the metric dist.

Proof. The proof combines well-known techniques from Žitković [26] and Delbaen and Schachermayer [11]. The details of how to modify these techniques to our space of controls $\mathscr{C}$ can be found in Theorem 3.3. in Kauppila [19].

The first step is to show that sets bounded in probability are convexly compact. Lemma A1.1 in [11] illustrates how a (generic) strictly concave functional on the space of interest (in our case the space of consumption plans) can be used to establish convergence of a subsequence of convex combinations. With minor modifications the technique can be used for nets as well.

The second part is to show that convexly compact sets are bounded in probability. Theorem 3.1 in Žitković [26] proves that closed and convex subsets of $L_{+}^{0}$ are convexly compact if and only if the set is bounded in probability. The "only if"-part of this theorem can be adapted to show that convexly compact subsets of the consumption space are bounded in probability.

We finish by noting a version of the common minimax theorem which uses convex compactness and follows with appropriate modifications from the basic outline of Theorem 3.1 in Simons [24]:

Theorem B.3. Let $\mathscr{A}$ be a nonempty convex, closed and convexly compact subset of a topological vector space and let $\mathscr{B}$ be a nonempty convex subset of another topological vector space. Let furthermore

$$
\begin{aligned}
\mathbb{H}: \mathscr{A} \times \mathscr{B} & \rightarrow(-\infty, \infty) \\
(A, B) & \mapsto \mathbb{H}(A, B)
\end{aligned}
$$

be concave and upper-semicontinuous in $A \in \mathscr{A}$ for $B \in \mathscr{B}$ fixed, and convex in $B \in \mathscr{B}$ for $A \in \mathscr{A}$ fixed.

Then we have the minimax relation

$$
\sup _{A \in \mathscr{A}} \inf _{B \in \mathscr{B}} \mathbb{H}(A, B)=\inf _{B \in \mathscr{B}} \sup _{A \in \mathscr{A}} \mathbb{H}(A, B) .
$$

Proof. It is easy to see that " $\leq$ " holds true in (58). For the proof of " $\geq$ " we let $\alpha \triangleq \inf _{B \in \mathscr{B}} \sup _{A \in \mathscr{A}} \mathbb{H}(A, B)$ and we will show that

$$
\{A \in \mathscr{A}: \mathbb{H}(A, B) \geq \alpha\}, \quad B \in \mathscr{B},
$$


is a collection of closed convex subsets of $\mathscr{A}$ which satisfies the finite intersection property. Convex compactness of $\mathscr{A}$ then implies that

$$
\bigcap_{B \in \mathscr{B}}\{A \in \mathscr{A}: \mathbb{H}(A, B) \geq \alpha\} \neq \emptyset
$$

i.e., there is $A^{*} \in \mathscr{A}$ such that $\inf _{B \in \mathscr{B}} \mathbb{H}\left(A^{*}, B\right) \geq \alpha$ and, thus, " $\geq$ " must hold in (58) as claimed.

By upper-semicontinuity and concavity of $\mathbb{H}$ in its first variable, each of the level sets $\{A \in \mathscr{A}: \mathbb{H}(A, B) \geq \alpha\}, B \in \mathscr{B}$, is closed and convex. To prove the finite intersection property consider $B_{1}, \ldots, B_{m} \in \mathscr{B}$ and observe that by the Mazur-Orlicz Theorem (Lemma 2.1 (b) in [24]) there are weights $\lambda_{1}, \ldots, \lambda_{m} \geq 0$ with $\sum_{i=1}^{m} \lambda_{i}=1$ such that

$$
\sup _{A \in \mathscr{A}}\left\{\mathbb{H}\left(A, B_{1}\right) \wedge \cdots \wedge \mathbb{H}\left(A, B_{m}\right)\right\}=\sup _{A \in \mathscr{A}}\left\{\lambda_{1} \mathbb{H}\left(A, B_{1}\right)+\cdots+\lambda_{m} \mathbb{H}\left(A, B_{m}\right)\right\}
$$

By assumption $\mathbb{H}(A,$.$) is convex for any A \in \mathscr{A}$ and so the preceding identity entails

$$
\left.\sup _{A \in \mathscr{A}}\left\{\mathbb{H}\left(A, B_{1}\right) \wedge \cdots \wedge \mathbb{H}\left(A, B_{m}\right)\right\} \geq \sup _{A \in \mathscr{A}} \mathbb{H}\left(A, \lambda_{1} B_{1}+\cdots+\lambda_{m} B_{m}\right)\right] \geq \alpha
$$

The finite intersection property thus follows once we have shown that the first supremum is actually attained. So let $\mathbb{H}^{\wedge}(A) \triangleq \mathbb{H}\left(A, B_{1}\right) \wedge \cdots \wedge$ $\mathbb{H}\left(A, B_{m}\right), A \in \mathscr{A}$, and consider a maximizing sequence $A_{1}, A_{2}, \cdots \in \mathscr{A}$ for $\sup _{A \in \mathscr{A}} \mathbb{H}^{\wedge}(A)$. Because $\mathscr{A}$ is convexly compact there is a convergent subnet of finite convex combinations, i.e., there is a convergent net $\left(A_{e}\right)_{e \in E}$ of $A_{e}=\sum_{n} \gamma_{n}^{e} A_{n}$ with $\gamma_{n}^{e}=0$ for $n \geq N_{e}$ and $\sum_{n} \gamma_{n}^{e}=1$ such that, in addition, for any $N=1,2, \ldots$ there is an $e_{N} \in E$ with $\gamma_{n}^{e}=0, n=0, \ldots, N$, for any $e \succeq e_{N}$; see Žitković [26], Definition 2.3. By concavity of $\mathbb{H}$ with respect to its first variable, also $\mathbb{H}^{\wedge}$ is concave and so

$$
\mathbb{H}^{\wedge}\left(A_{e}\right) \geq \sum_{n} \gamma_{n}^{e} \mathbb{H}^{\wedge}\left(A_{n}\right)
$$

The upper-semicontinuity of $\mathbb{H}$ in its first variable entails the upper-semicontinuity of $\mathbb{H}^{\wedge}$. This allows us to conclude in the limit that $A_{0} \triangleq \lim _{e \in E} A_{e} \in$ $\mathscr{A}$ attains $\sup _{A \in \mathscr{A}} \mathbb{H}^{\wedge}(A)$. 


\section{References}

[1] Luis H.R. Alvarez. Optimal capital accumulation under price uncertainty and costly reversibility. Journal of Economic Dynamics 86 Control, 35:1769-1788, 2011.

[2] Peter Bank and Christoph Baumgarten. Parameter-dependent optimal stopping problems for one-dimensional diffusions. Electron. J. Probab., 15:no. 64, 1971-1993, 2010. ISSN 1083-6489. doi: 10.1214/EJP.v15-835. URL http://dx.doi.org/10.1214/EJP.v15-835.

[3] Peter Bank and Nicole El Karoui. A stochastic representation theorem with applications to optimization and obstacle problems. Ann. Probab., 32(1B):1030-1067, 2004.

[4] Peter Bank and Hans Föllmer. American options, multi-armed bandits, and optimal consumption plans: a unifying view. In Paris-Princeton Lectures on Mathematical Finance, 2002, volume 1814 of Lecture Notes in Math., pages 1-42. Springer, Berlin, 2003.

[5] Peter Bank and Frank Riedel. Optimal consumption choice with intertemporal substitution. Ann. Appl. Probab., 11(3):750-788, 2001. ISSN 1050-5164.

[6] V. E. Beneš, L. A. Shepp, and H. S. Witsenhausen. Some solvable stochastic control problems. Stochastics, 4(1):39-83, 1980/81. ISSN 0090-9491. doi: 10.1080/17442508008833156. URL http://dx.doi.org/10.1080/17442508008833156.

[7] Fred Espen Benth, Kenneth Hvistendahl Karlsen, and Kristin Reikvam. Optimal portfolio management rules in a non-Gaussian market with durability and intertemporal substitution. Finance Stoch., 5(4):447-467, 2001. ISSN 0949-2984.

[8] Bruno Bouchard and Huyên Pham. Wealth-path dependent utility maximization in incomplete markets. Finance Stoch., 8(4):579 603, 2004. ISSN 0949-2984. doi: 10.1007/s00780-004-0125-8. URL http://dx.doi.org/10.1007/s00780-004-0125-8. 
[9] Abel Cadenillas and Ulrich G. Haussmann. The stochastic maximum principle for a singular control problem. Stochastics Stochastics Rep., 49(3-4):211-237, 1994. ISSN 1045-1129.

[10] Maria B. Chiarolla and Giorgio Ferrari. Identifying the free boundary of a stochastic, irreversible investment problem via the BankEl Karoui representation theorem. SIAM J. Control Optim., 52(2): 1048-1070, 2014. ISSN 0363-0129. doi: 10.1137/11085195X. URL http://dx.doi.org/10.1137/11085195X.

[11] Freddy Delbaen and Walter Schachermayer. A general version of the fundamental theorem of asset pricing. Math. Ann., 300(3):463-520, 1994. ISSN 0025-5831.

[12] Freddy Delbaen and Walter Schachermayer. The fundamental theorem of asset pricing for unbounded stochastic processes. Math. Ann., 312(2): 215-250, 1998. ISSN 0025-5831.

[13] Giorgio Ferrari. On an integral equation for the free-boundary of stochastic, irreversible investment problems. The Annals of Applied Probability, forthcoming.

[14] Ayman Hindy and Chi-fu Huang. Intertemporal preferences for uncertain consumption: a continuous-time approach. Econometrica, 60: 781-801, 1992.

[15] Ayman Hindy and Chi-fu Huang. Optimal consumption and portfolio rules with durability and local substitution. Econometrica, 61:85-121, 1993.

[16] Ayman Hindy, Chi-fu Huang, and David Kreps. On intertemporal preferences in continuous time: the case of certainty. J. Math. Econom., 21 (5):401-440, 1992. ISSN 0304-4068.

[17] Ioannis Karatzas and Steven E. Shreve. Connections between optimal stopping and singular stochastic control. I. Monotone follower problems. SIAM J. Control Optim., 22(6):856-877, 1984. ISSN 0363-0129. doi: 10.1137/0322054. URL http://dx.doi.org/10.1137/0322054. 
[18] Ioannis Karatzas and Gordan Žitković. Optimal consumption from investment and random endowment in incomplete semimartingale markets. Ann. Probab., 31(4):1821-1858, 2003. ISSN 0091-1798.

[19] Helena Kauppila. Convex duality in singular control - optimal consumption choice with intertemporal substitution and optimal investment in incomplete markets. PhD thesis, Columbia University, 2010.

[20] D. Kramkov and W. Schachermayer. The asymptotic elasticity of utility functions and optimal investment in incomplete markets. Ann. Appl. Probab., 9(3):904-950, 1999. ISSN 1050-5164.

[21] D. Kramkov and W. Schachermayer. Necessary and sufficient conditions in the problem of optimal investment in incomplete markets. Ann. Appl. Probab., 13(4):1504-1516, 2003. ISSN 1050-5164.

[22] Robert C. Merton. Optimum consumption and portfolio rules in a continuous-time model. J. Econom. Theory, 3(4):373-413, 1971. ISSN 0022-0531.

[23] R. Tyrrell Rockafellar. Convex analysis. Princeton Mathematical Series, No. 28. Princeton University Press, Princeton, N.J., 1970.

[24] Stephen Simons. Minimax and monotonicity, volume 1693 of Lecture Notes in Mathematics. Springer-Verlag, Berlin, 1998. ISBN 3-540-647554.

[25] Gordan Žitković. Utility maximization with a stochastic clock and an unbounded random endowment. Ann. Appl. Probab., 15(1B):748-777, 2005. ISSN 1050-5164.

[26] Gordan Žitković. Convex compactness and its applications. Math. Financ. Econ., 3(1):1-12, 2010. ISSN 1862-9679. doi: 10.1007/s11579-010-0024-z. URL http://dx.doi.org/10.1007/s11579-010-0024-z. 